\title{
An Improved Cu-Based Catalyst System for the Reactions of Alcohols with Aryl Halides
}

\author{
Ryan A. Altman, Alex Shafir, Alice Choi, Philip A. Lichtor, and Stephen L. Buchwald* \\ Department of Chemistry, Massachusetts Institute of Technology
}

Cambridge, MA 02139

sbuchwal@mit.edu

\section{Supporting Information}

\section{Table of Contents:}

General Considerations

General procedure for the $\mathrm{Cu}$-catalyzed cross-coupling of alcohols with aryl halides

References 


\section{General Considerations}

All reactions were carried out in resealable test tubes with teflon septa under a dry argon or nitrogen atmosphere. Copper(I) iodide (98\%) was purchased from Strem. $\mathrm{Me}_{4} \mathrm{Phen}$ was purchased from Acros. The Anhydrous finely powdered $\mathrm{Cs}_{2} \mathrm{CO}_{3}$ was a generous gift from Chemetall. This base was stored under nitrogen in a Vacuum Atmospheres glovebox. (The base is hygroscopic and excessive amounts of water lead to the formation of phenol and diaryl ether byproducts.) Small portions of the base ( $\sim 5 \mathrm{~g})$ were removed from the glovebox in glass vials, stored in the air in a desiccator filled with anhydrous calcium sulfate, and weighed in the air. Alcohols were purchased from commercial sources and used without further purification. Aryl halides were purchased from commercial sources and, when necessary, filtered through neutral alumina or distilled. Anhydrous toluene was purchased from J. T. Baker in CYCLE-TRAINER® solvent delivery kegs and vigorously purged with argon for $2 \mathrm{~h}$. The solvent was further purified by passing it through two packed columns of neutral alumina under argon. The solvents were transferred by syringe from the solvent purification system to the reaction flask. Flash column chromatography was performed using a Biotage SP4 Flash Purification System using KP-Sil silica cartridges. In all cases, dichloromethane was used to transfer the crude reaction material onto the silica gel samplet. The samplet was dried in an oven prior to usage. A gradient elution using hexane and ethyl acetate was performed, based on the recommendation from the Biotage TLC Wizard.

Unless specified, yields reported in the publication are of the isolated material and represent an average of at least two independent runs. Yields reported in the supporting information refer to a single experiment. Compounds described in the literature were characterized by comparing their ${ }^{1} \mathrm{H}$ NMR and ${ }^{13} \mathrm{C}$ NMR spectra, and melting points (m.p.) to 
the previously reported data; their purity was confirmed by gas chromatography (GC) or elemental analysis. GC analyses were performed on a Hewlett Packard 6890 instrument with an FID detector and a Hewlett Packard 10 m x 0.2 mm i.d. HP-1 capillary column using dodecane as an internal standard. Elemental analyses were performed by Atlantic Microlabs, Inc., Norcross, GA. Previously unknown compounds were synthesized, purified and analyzed from a single run and were then repeated to determine an average yield. They were characterized by ${ }^{1} \mathrm{H}$ NMR, ${ }^{13} \mathrm{C}$ NMR, m.p., IR and elemental analysis. For those compounds that did not give a satisfactory elemental analysis, a copy of their ${ }^{1} \mathrm{H}$ NMR spectra is included. ${ }^{1} \mathrm{H}$ NMR and ${ }^{13} \mathrm{C}$ NMR spectra were recorded on Varian $500 \mathrm{MHz}$ instruments with chemical shifts reported relative to the deuterated solvent or TMS. IR spectra were recorded on a Perkin-Elmer System 2000 FT-IR instrument for all previously unknown compounds $(\mathrm{KBr}$ disc). Melting points (uncorrected) were obtained on a Mel-Temp II capillary melting point apparatus.

\section{General procedure for the $\mathrm{Cu}$-catalyzed cross-coupling of alcohols with aryl halides}

An oven-dried screw-cap test tube was charged with $\mathrm{CuI}(9.5 \mathrm{mg}, 0.050 \mathrm{mmol}), \mathrm{Me}_{4}$ Phen (24 $\mathrm{mg}, 0.10 \mathrm{mmol})$, aryl halide $\left(1.0 \mathrm{mmol}\right.$, if solid), $\mathrm{Cs}_{2} \mathrm{CO}_{3}(490 \mathrm{mg}, 1.5 \mathrm{mmol})$, and a magnetic stir bar. The reaction vessel was fitted with a rubber septum. The test tube was evacuated and back-filled with dry argon. Aryl halide $(1.0 \mathrm{mmol}$, if liquid), and toluene $(0.50 \mathrm{~mL})$ were then added by syringe. The rubber septum was removed and the reaction tube was quickly sealed with a Teflon-lined septum. The vessel was immersed in a pre-heated oil bath and stirred vigorously until TLC and/or GC analysis of the crude reaction mixture indicated that the aryl halide had been completely consumed. The reaction mixture was cooled to room temperature, diluted with ethyl acetate $(15 \mathrm{~mL})$, and filtered through a plug of silica, eluting with additional ethyl acetate 
(30 $\mathrm{mL})$. The filtrate was concentrated and the resulting residue was purified by flash chromatography (hexane/ethyl acetate) to provide the desired product.

\section{Experimental procedures for compounds in Table 2}<smiles>CCCCCOc1ccc(OC)cc1</smiles>

4-(hexyloxy)-anisole (Entries 1-3)

The general procedure was followed using $\mathrm{CuI}(9.5 \mathrm{mg}, 0.050 \mathrm{mmol}), \mathrm{Me}_{4} \mathrm{Phen}(24 \mathrm{mg}, 0.10$ mmol), $\mathrm{Cs}_{2} \mathrm{CO}_{3}(391 \mathrm{mg}, 1.2 \mathrm{mmol})$, 4-iodoanisole (234 mg, $\left.1.00 \mathrm{mmol}\right)$, and $n$-hexanol (186 $\mu \mathrm{L}, 1.50 \mathrm{mmol})$ with toluene $(0.50 \mathrm{~mL})$ as solvent for $15 \mathrm{~h}$ at $80{ }^{\circ} \mathrm{C}$. After cooling to room temperature, dodecane $(225 \mathrm{~mL}, 1.0 \mathrm{mmol})$ and ethyl acetate $(20 \mathrm{~mL})$ were stirred into the reaction mixture. The mixture was filtered through a small plug of silica gel, and sampled for GC analysis. In order to standardize this compound for GC analysis, the product was purified by flash chromatography (hexane / ethyl acetate 1:0 $\rightarrow$ 9:1) to afford the title compound as a colorless oil (162 mg, 78\%). ${ }^{1} \mathrm{H}$ NMR $\left(300 \mathrm{MHz}, \mathrm{CDCl}_{3}\right) \delta 6.85(4 \mathrm{H}, \mathrm{s}), 3.94-3.89(3 \mathrm{H}, \mathrm{t}, J=$ $6.6 \mathrm{~Hz}), 3.78(3 \mathrm{H}, \mathrm{s}), 1.79-1.72(2 \mathrm{H}, \mathrm{m}), 1.49-1.37(2 \mathrm{H}, \mathrm{m}), 1.36-1.32(4 \mathrm{H}, \mathrm{m}), 0.94-0.90(3 \mathrm{H}, \mathrm{t}$, $J=7.0 \mathrm{~Hz}) .{ }^{13} \mathrm{C}$ NMR $\left(125 \mathrm{MHz}, \mathrm{CDCl}_{3}\right) \delta 153.9,153.5,115.6,115.0,68.9,56.0,31.9,29.6$, 26.0, 22.9, 14.3. IR (KBr disc, $\left.\mathrm{cm}^{-1}\right)$ 2937, 1510, 1466, 1290, 1235, 1113, 1036, 827, 726, 532. Anal. Calc. for $\mathrm{C}_{13} \mathrm{H}_{20} \mathrm{O}_{2}$ : C 74.96, H 9.68. Found: C 74.78, H 9.74.

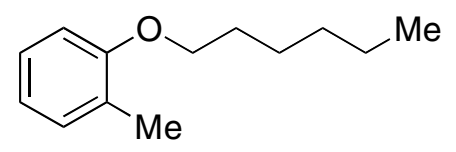

2-(hexyloxy)-toluene (Entry 4) ${ }^{1}$

The general procedure was followed using $\mathrm{CuI}(9.5 \mathrm{mg}, 0.050 \mathrm{mmol}), \mathrm{Me}_{4} \mathrm{Phen}(24 \mathrm{mg}, 0.10$ 
mmol), $\mathrm{Cs}_{2} \mathrm{CO}_{3}$ (490 mg, $\left.1.5 \mathrm{mmol}\right), 2$-iodotoluene (127 $\left.\mu \mathrm{L}, 1.00 \mathrm{mmol}\right)$, and $n$-hexanol (187 $\mu \mathrm{L}, 1.50 \mathrm{~mol})$ with toluene $(0.50 \mathrm{~mL})$ as solvent for $24 \mathrm{~h}$ at $110{ }^{\circ} \mathrm{C}$. Chromatographic purification (hexane / ethyl acetate 1:0 $\rightarrow$ 9:1) afforded the title compound as a clear oil (159 mg, 83\%). ${ }^{1} \mathrm{H}$ NMR (500 MHz, $\left.\mathrm{CDCl}_{3}\right) \delta$ 7.16-7.12 (2H, m), 6.85-6.80 (2H, m), $3.96(2 \mathrm{H}, \mathrm{t}, J=$ $6.4 \mathrm{~Hz}), 2.23(3 \mathrm{H}, \mathrm{s}), 1.85-1.76(2 \mathrm{H}, \mathrm{m}), 1.56-1.32(6 \mathrm{H}, \mathrm{m}), 0.94-0.88(3 \mathrm{H}, \mathrm{m}) .{ }^{13} \mathrm{C}$ NMR $(125$ $\left.\mathrm{MHz}, \mathrm{CDCl}_{3}\right) \delta 157.6,130.9,127.1,127.0,120.3,111.1,68.1,32.0,29.7,26.2,23.0,16.6,14.4$. IR (KBr disc, $\left.\mathrm{cm}^{-1}\right)$ 2955, 2931, 2860, 1603, 1496, 1463, 1379, 1245, 119, 1122, 1050, 749, 713. Anal. Calc. for $\mathrm{C}_{13} \mathrm{H}_{20} \mathrm{O}$ : C 81.20, H 10.48. Found: C 81.41, H 10.33.

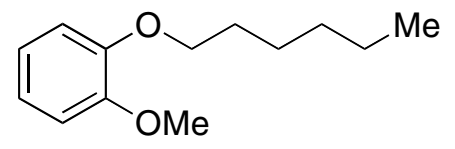

2-(hexyloxy)-anisole (Entry 5) ${ }^{\mathrm{i}}$

The general procedure was followed using $\mathrm{CuI}(9.5 \mathrm{mg}, 0.050 \mathrm{mmol}), \mathrm{Me}_{4} \mathrm{Phen}(24 \mathrm{mg}, 0.10$ $\mathrm{mmol}), \mathrm{Cs}_{2} \mathrm{CO}_{3}(490 \mathrm{mg}, 1.5 \mathrm{mmol}), 2$-iodoanisole $(130 \mu \mathrm{L}, 1.00 \mathrm{mmol})$, and $n$-hexanol (187 $\mu \mathrm{L}, 1.50 \mathrm{~mol})$ with toluene $(0.50 \mathrm{~mL})$ as solvent for $30 \mathrm{~h}$ at $110{ }^{\circ} \mathrm{C}$. Chromatographic purification (hexane / ethyl acetate 1:0 $\rightarrow$ 9:1) afforded the title compound as a clear oil (195 mg, 94\%). ${ }^{1} \mathrm{H}$ NMR (500 MHz, $\left.\mathrm{CDCl}_{3}\right) \delta$ 6.92-6.90 (4H, m), $4.03(2 \mathrm{H}, \mathrm{t}, J=7.0 \mathrm{~Hz}), 3.88(3 \mathrm{H}$, s), $1.86(2 \mathrm{H}, \mathrm{m}), 7.49-1.34(8 \mathrm{H}, \mathrm{m}), 0.93-0.90(3 \mathrm{H}, \mathrm{m}) .{ }^{13} \mathrm{C} \mathrm{NMR}\left(125 \mathrm{MHz}, \mathrm{CDCl}_{3}\right) \delta 149.6$ 148.8, 121.0, 121.0, 113.2, 112.0, 69.2, 56.2, 31.9, 29.4, 25.9, 22.8, 14.3. IR (KBr disc, $\left.\mathrm{cm}^{-1}\right)$ 2932, 2860, 1593, 1507, 1456, 1253, 1228, 1180, 1125, 1030, 740. Anal. Calc. for $\mathrm{C}_{13} \mathrm{H}_{20} \mathrm{O}_{2}$ : C 74.96, H 9.68. Found: C 74.69, H 9.68.<smiles>CCCCCCOc1ccccc1Cl</smiles>

1-chloro-2-(hexyloxy)benzene (Entry 6) ${ }^{\mathrm{ii}}$ 
The general procedure was followed using $\mathrm{CuI}(9.5 \mathrm{mg}, 0.050 \mathrm{mmol}), \mathrm{Me}_{4}$ Phen $(24 \mathrm{mg}, 0.10$ $\mathrm{mmol}), \mathrm{Cs}_{2} \mathrm{CO}_{3}(490 \mathrm{mg}, 1.5 \mathrm{mmol}), 1$-chloro-2-iodobenzene $(122 \mu \mathrm{L}, 1.00 \mathrm{mmol})$, and $n$ hexanol $(187 \mu \mathrm{L}, 1.50 \mathrm{~mol})$ with toluene $(0.50 \mathrm{~mL})$ as solvent for $24 \mathrm{~h}$ at $110{ }^{\circ} \mathrm{C}$. Chromatographic purification (hexane / ethyl acetate 1:0 $\rightarrow$ 1:3) afforded the title compound as a clear oil (185 mg, 87\%). ${ }^{1} \mathrm{H} \mathrm{NMR}\left(500 \mathrm{MHz}, \mathrm{CDCl}_{3}\right) \delta 7.36(1 \mathrm{H}, \mathrm{dd}, J=1.7,7.9 \mathrm{~Hz}), 7.20(1 \mathrm{H}$, m), 6.94-6.85 (2H, m), $4.03(2 \mathrm{H}, \mathrm{t}, J=6.5 \mathrm{~Hz}), 1.85(2 \mathrm{H}, \mathrm{m}), 1.54-1.31(\mathrm{~m}, 6 \mathrm{H}), 0.94-0.89(\mathrm{~m}$, $3 \mathrm{H})$.<smiles>COc1ccc(OC)cc1</smiles>

1,4-dimethoxybenzene (Entry 7$)^{\text {iii }}$

The general procedure was followed using $\mathrm{CuI}(9.5 \mathrm{mg}, 0.050 \mathrm{mmol}), \mathrm{Me}_{4} \mathrm{Phen}(24 \mathrm{mg}, 0.10$ $\mathrm{mmol}), \mathrm{Cs}_{2} \mathrm{CO}_{3}(391 \mathrm{mg}, 1.2 \mathrm{mmol})$, 4-iodoanisole $(234 \mathrm{mg}, 1.00 \mathrm{mmol})$, and methanol $(81 \mu \mathrm{L}$, $2.00 \mathrm{mmol})$ with toluene $(0.50 \mathrm{~mL})$ as solvent for $15 \mathrm{~h}$ at $80{ }^{\circ} \mathrm{C}$. Chromatographic purification (hexane / ethyl acetate 1:0 $\rightarrow$ 9:1) afforded the title compound as a colorless oil (108 mg, 78\%). ${ }^{1} \mathrm{H}$ NMR (300 MHz, $\left.\mathrm{CDCl}_{3}\right) \delta 6.84$ (4H, s), 3.77 (6H, s). IR (KBr disc, $\left.\mathrm{cm}^{-1}\right)$ 2933, 2860, 1509, $1467,1233,1181,1107,1042,824,742,724,523$.<smiles>CCOc1ccc(Br)cc1</smiles>

1-bromo-4-ethoxybenzene $(\text { Entry } 8)^{\text {iv }}$

The general procedure was followed using $\mathrm{CuI}(9.5 \mathrm{mg}, 0.050 \mathrm{mmol}), \mathrm{Me}_{4} \mathrm{Phen}(24 \mathrm{mg}, 0.10$ $\mathrm{mmol}$ ), $\mathrm{Cs}_{2} \mathrm{CO}_{3}$ (490 mg, $1.5 \mathrm{mmol}$ ), 1,bromo-4-iodobenzene (283 mg, $1.00 \mathrm{mmol}$ ), and ethanol $(116 \mu \mathrm{L}, 2.0 \mathrm{~mol})$ with toluene $(0.50 \mathrm{~mL})$ as solvent for $24 \mathrm{~h}$ at $80{ }^{\circ} \mathrm{C}$. Chromatographic purification (hexane / ethyl acetate 1:0 $\rightarrow$ 9:1) afforded the title compound as a clear oil (130 
$\mathrm{mg}, 65 \%) .{ }^{1} \mathrm{H}$ NMR $\left(500 \mathrm{MHz}, \mathrm{CDCl}_{3}\right) \delta$ 7.39-7.36 (2H, m), 6.80-6.77 (2H, m), $4.00(2 \mathrm{H}, \mathrm{q}, J=$ $7.0 \mathrm{~Hz}), 1.42(\mathrm{t}, 3 \mathrm{H}, J=7.0 \mathrm{~Hz}) .{ }^{13} \mathrm{C} \mathrm{NMR}\left(125 \mathrm{MHz}, \mathrm{CDCl}_{3}\right) \delta 158.2,132.4,116.4,112.8$, 63.9, 14.9. IR ( $\mathrm{KBr}$ disc, $\left.\mathrm{cm}^{-1}\right)$ 2981, 2927, 1592, 1579, 1489, 1475, 1393, 1286, 1245, 1172 , 1115, 1072, 1048, 1002, 923, 820, 639, 507. Anal. Calc. for $\mathrm{C}_{8} \mathrm{H}_{9} \mathrm{BrO}$ : C 47.79, H 4.51. Found: C 47.62, H 4.55.<smiles>CC1(C)OCC(COc2ccc(Cl)cc2)O1</smiles>

4-((4-chlorophenoxy)methyl)-2,2-dimethyl-1,3-dioxolane (Entry 9)

The general procedure was followed using $\mathrm{CuI}(9.5 \mathrm{mg}, 0.050 \mathrm{mmol}), \mathrm{Me}_{4} \mathrm{Phen}(24 \mathrm{mg}, 0.10$ $\mathrm{mmol}), \mathrm{Cs}_{2} \mathrm{CO}_{3}(650 \mathrm{mg}, 2.0 \mathrm{mmol}), 4$-chloro-1-iodobenzene (238 mg, $\left.1.00 \mathrm{mmol}\right)$, and solketal $(249 \mu \mathrm{L}, 2.00 \mathrm{mmol})$ with toluene $(0.50 \mathrm{~mL})$ as solvent for $15 \mathrm{~h}$ at $80{ }^{\circ} \mathrm{C}$. Chromatographic purification (hexane / ethyl acetate 1:0 $\rightarrow$ 9:1) afforded the title compound as a colorless oil (202 $\mathrm{mg}, 83 \%) .{ }^{1} \mathrm{H}$ NMR $\left(300 \mathrm{MHz}, \mathrm{CDCl}_{3}\right) \delta$ 7.268-7.209 (2H, m), 6.872-6.819 (2H, m), 4.512$4.434(1 \mathrm{H}, \mathrm{m}), 4.192-4.143(1 \mathrm{H}, \mathrm{dd}, J=6.4,8.5 \mathrm{~Hz}), 4.046-3.996(1 \mathrm{H}, \mathrm{dd}, J=5.5,9.5 \mathrm{~Hz})$, 3.938-3.868 (2H, m), $1.465(3 \mathrm{H}, \mathrm{s}), 1.407(3 \mathrm{H}, \mathrm{s}) .{ }^{13} \mathrm{C} \mathrm{NMR}\left(125 \mathrm{MHz}, \mathrm{CDCl}_{3}\right) \delta 157.3,129.5$, 126.2, 116.0, 110.0, 74.1, 69.2, 66.9, 27.0, 25.5. IR (KBr disc, $\left.\mathrm{cm}^{-1}\right)$ 2980, 2932, 1489, 1451, 1371, 1240, 1203, 1169, 1152, 1075, 1051, 1000, 973, 893, 831, 658. Anal. Calc. for $\mathrm{C}_{12} \mathrm{H}_{15} \mathrm{ClO}_{3}$ : C 59.39, H 6.23. Found: C 59.57, H 6.30.<smiles>CC=CCOc1ccc(F)cc1</smiles>

(E)-1-(but-2-enyloxy)-4-fluorobenzene (Entry 10) ${ }^{\mathrm{v}}$

The general procedure was followed using $\mathrm{CuI}(9.5 \mathrm{mg}, 0.050 \mathrm{mmol}), \mathrm{Me}_{4} \mathrm{Phen}(24 \mathrm{mg}, 0.10$ 
$\mathrm{mmol}), \mathrm{Cs}_{2} \mathrm{CO}_{3}(490 \mathrm{mg}, 1.5 \mathrm{mmol}), 1$-fluoro-4-iodobenzene (115 $\left.\mu \mathrm{L}, 1.00 \mathrm{mmol}\right)$, and (E)crotyl alcohol $(127 \mu \mathrm{L}, 1.50 \mathrm{~mol})$ with toluene $(0.50 \mathrm{~mL})$ as solvent for $24 \mathrm{~h}$ at $80{ }^{\circ} \mathrm{C}$. Chromatographic purification (hexane / ethyl acetate 1:0 $\rightarrow$ 9:1) afforded the title compound as a clear oil (117 mg, 70\%). ${ }^{1} \mathrm{H}$ NMR (500 MHz, $\left.\mathrm{CDCl}_{3}\right) \delta$ 7.00-6.95 (2H, m), 6.89-6.83 (2H, m), 5.90-5.83 (1H, m), 5.75-5.70 (1H, m), $4343(2 \mathrm{H}, \mathrm{dd}, J=0.9,6.2 \mathrm{~Hz}), 1.77(3 \mathrm{H}, \mathrm{d}, J=6.4 \mathrm{~Hz})$. ${ }^{13} \mathrm{C}$ NMR $\left(125 \mathrm{MHz}, \mathrm{CDCl}_{3}\right) \delta 156.4,155.0,130.9,126.1,116.0,115.8,69.5,18.1 . \mathrm{IR}(\mathrm{KBr}$ disc $\mathrm{cm}^{-1}$ ) 3025, 2941, 2919, 2859, 1506, 1463, 1379, 1293, 1246, 1208, 1097, 1009, 967, 828, 780, 741, 514. Anal. Calc. for $\mathrm{C}_{10} \mathrm{H}_{11} \mathrm{FO}$ : C 72.27, H 6.67. Found: C 72.00, H 6.81.<smiles>Nc1ccc(OCc2ccccc2)cc1</smiles>

4-(benzyloxy)aniline (Entry 11) $)^{\mathrm{vi}}$

The general procedure was followed using $\mathrm{CuI}(9.5 \mathrm{mg}, 0.050 \mathrm{mmol}), \mathrm{Me}_{4} \mathrm{Phen}(24 \mathrm{mg}, 0.10$ mmol), $\mathrm{Cs}_{2} \mathrm{CO}_{3}(391 \mathrm{mg}, 1.2 \mathrm{mmol}$ ), 4-iodoaniline (219 mg, $1.00 \mathrm{mmol}$ ), and benzyl alcohol $(210 \mu \mathrm{L}, 2.00 \mathrm{mmol})$ with toluene $(0.50 \mathrm{~mL})$ as solvent for $15 \mathrm{~h}$ at $80^{\circ} \mathrm{C}$. Chromatographic purification (hexane / ethyl acetate 1:0 $\rightarrow$ 9:1) afforded the title compound as a red solid (158 mg, 79\%). ${ }^{1} \mathrm{H}$ NMR (300 MHz, $\left.\mathrm{CDCl}_{3}\right) \delta$ 7.45-7.32 (5H, m), 6.86-6.80 (2H, m), 6.68-6.63 (2H,m), $3.428(2 \mathrm{H}, \mathrm{b} \mathrm{s}) .{ }^{13} \mathrm{C}$ NMR (125 MHz, $\left.\mathrm{CDCl}_{3}\right) \delta 152.1,140.4,137.7,128.7,128.0,127.7$, 116.5, 116.2, 70.9. IR (KBr disc, $\left.\mathrm{cm}^{-1}\right)$ 2932, 2960, 1585, 1568, 1462, 1382, 1274, 1228, 1127 , 1109, 1014, 829, 727, 673, 627, 423. m.p. $45-46.5^{\circ} \mathrm{C}$.

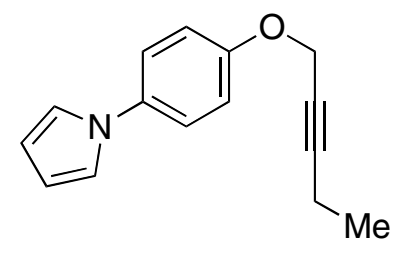


1-(4-(pent-2-ynyloxy)phenyl)-1H-pyrrole (Entry 12)

The general procedure was followed using $\mathrm{CuI}(9.5 \mathrm{mg}, 0.050 \mathrm{mmol}), \mathrm{Me}_{4} \mathrm{Phen}(24 \mathrm{mg}, 0.10$ $\mathrm{mmol}), \mathrm{Cs}_{2} \mathrm{CO}_{3}(391 \mathrm{mg}, 1.2 \mathrm{mmol}), 1$-(4-iodophenyl)pyrrole (269 mg, $\left.1.00 \mathrm{mmol}\right)$, and 2pentyn-1-ol $(139 \mu \mathrm{L}, 1.50 \mathrm{mmol})$ with toluene $(0.50 \mathrm{~mL})$ as solvent for $24 \mathrm{~h}$ at $80{ }^{\circ} \mathrm{C}$. Chromatographic purification (hexane / ethyl acetate 1:0 $\rightarrow$ 9:1) afforded the title compound as a white solid (209 mg, 92\%). ${ }^{1} \mathrm{H}$ NMR (300 MHz, $\left.\mathrm{CDCl}_{3}\right) \delta$ 7.35-7.30 (2H, m), 7.06-7.01 (4H, m), 6.34-6.33 (2H, t, $J=2.2 \mathrm{~Hz}), 4.71-4.70(2 \mathrm{H},, J=2.2 \mathrm{~Hz}), 2.31-2.22(2 \mathrm{H}, \mathrm{m}), 1.19-1.14(3 \mathrm{H}, \mathrm{t}, J$ $=7.5 \mathrm{~Hz}) .{ }^{13} \mathrm{C} \mathrm{NMR}\left(125 \mathrm{MHz}, \mathrm{CDCl}_{3}\right) \delta 156.0,122.2,119.9,115.9,110.1,90.0,74.1,57.0$, 44.8, 13.8, 12.7. IR (KBr disc, $\left.\mathrm{cm}^{-1}\right)$ 3132, 2977, 1522, 1325, 1258, 1243, 1190, 1070, 1018, 1006, 920, 824, 734. m.p. 57.5-59.0 ${ }^{\circ} \mathrm{C}$.

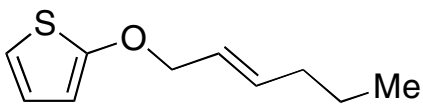

(E)-2-(hex-2-enyloxy)thiophene (Entry 13)

The general procedure was followed using $\mathrm{CuI}(9.5 \mathrm{mg}, 0.050 \mathrm{mmol}), \mathrm{Me}_{4} \mathrm{Phen}(24 \mathrm{mg}, 0.10$ mmol), $\mathrm{Cs}_{2} \mathrm{CO}_{3}(650 \mathrm{mg}, 2.0 \mathrm{mmol}), 3$-iodothiophene (102 $\left.\mu \mathrm{L}, 1.00 \mathrm{mmol}\right)$, and $n$-hexanol (236 $\mu \mathrm{L}, 2.00 \mathrm{mmol})$ with toluene $(0.50 \mathrm{~mL})$ as solvent for $15 \mathrm{~h}$ at $80{ }^{\circ} \mathrm{C}$. Chromatographic purification (hexane / ethyl acetate $1: 0 \rightarrow 12.5: 1$ ) afforded the title compound as a yellow oil (125 mg, 69\%). ${ }^{1} \mathrm{H}$ NMR (300 MHz, $\left.\mathrm{CDCl}_{3}\right) \delta 7.191-7.163(1 \mathrm{H}, \mathrm{dd}, J=3.1,5.3 \mathrm{~Hz}), 6.789-$ $6.767(1 \mathrm{H}, \mathrm{dd}, J=1.6,5.3 \mathrm{~Hz}), 6.274-6.258(1 \mathrm{H}, \mathrm{q}, J=1.6 \mathrm{~Hz}), 5.906-5.811(1 \mathrm{H}, \mathrm{m}), 5.765-$ $5.666(1 \mathrm{H}, \mathrm{m}), 4.461-4.438(2 \mathrm{H}, \mathrm{dd}, J=1.0,6.0 \mathrm{~Hz}), 2.119-2.045(2 \mathrm{H}, \mathrm{m}), 1.507-1.384(2 \mathrm{H}, \mathrm{m})$, 0.951-0.902 (3H, t, $J=7.3 \mathrm{~Hz}) .{ }^{13} \mathrm{C}$ NMR $\left(125 \mathrm{MHz}, \mathrm{CDCl}_{3}\right) \delta 157.7,136.1,124.9,124.7$, 119.8, 97.6, 71.1, 34.6, 22.3, 13.9. IR ( $\mathrm{KBr}$ disc, $\left.\mathrm{cm}^{-1}\right)$ 3118, 2959, 2929, 2871, 1544, 1421, 1366, 1234, 1177, 1010, 970, 873, 831, 752, 627. Anal. Calc. for $\mathrm{C}_{10} \mathrm{H}_{14} \mathrm{OS}$ : C 65.89, $\mathrm{H} 7.74$. 
Found: C 66.05, H 7.92.<smiles>CCCCCCOc1ccc(C(=O)OC(C)(C)C)cc1</smiles>

tert-butyl 4-(hexyloxy)benzoate (Entry 14)

The general procedure was followed using $\mathrm{CuI}(9.5 \mathrm{mg}, 0.050 \mathrm{mmol}), \mathrm{Me}_{4} \mathrm{Phen}(24 \mathrm{mg}, 0.10$ mmol), $\mathrm{Cs}_{2} \mathrm{CO}_{3}\left(490 \mathrm{mg}, 1.5 \mathrm{mmol}\right.$ ), tert-butyl-4-iodo benzoate ${ }^{\mathrm{vii}}$ (304 mg, $1.00 \mathrm{mmol}$ ), and $n$ hexanol $(187 \mu \mathrm{L}, 1.50 \mathrm{~mol})$ with toluene $(0.50 \mathrm{~mL})$ as solvent for $24 \mathrm{~h}$ at $80{ }^{\circ} \mathrm{C}$. Chromatographic purification (hexane / ethyl acetate 1:0 $\rightarrow$ 19:1) afforded a mixture of the title compound and $n$-hexyl 4-(hexyloxy)benzoate (7:1 by ${ }^{1} \mathrm{H}$ NMR and GC) as a clear oil (264 mg, 95\%). ${ }^{1} \mathrm{H}$ NMR (500 MHz, $\left.\mathrm{CDCl}_{3}\right) \delta$ 7.98-7.92 (2H, m), 6.92-6.87 (2H, m), $4.00(2 \mathrm{H}, \mathrm{t}, J=6.6$ $\mathrm{Hz}), 1.83-1.76(2 \mathrm{H}, \mathrm{m}), 1.49-1.44(2 \mathrm{H}, \mathrm{m}), 1.35-1.30(2 \mathrm{H}, \mathrm{m}), 0.92(3 \mathrm{H}, \mathrm{m}) .{ }^{13} \mathrm{C}$ NMR $(125$ $\left.\mathrm{MHz}, \mathrm{CDCl}_{3}\right) \delta 165.9,162.8,131.5,124.4,114.0,80.6,68.3,31.8,29.3,28.5,25.9,22.8,14.2$. IR $\left(\mathrm{KBr}\right.$ disc, $\left.\mathrm{cm}^{-1}\right)$ 2933, 2872, 1710, 1607, 1510, 1368, 1293, 1253, 1160, 1116, 1010, 848, 771, 696.<smiles>CCCCCCOc1ccc(Cl)nc1</smiles>

2-chloro-5-(hexyloxy)pyridine (Entry 15)

The general procedure was followed using $\mathrm{CuI}(9.5 \mathrm{mg}, 0.050 \mathrm{mmol}), \mathrm{Me}_{4} \mathrm{Phen}(24 \mathrm{mg}, 0.10$ $\mathrm{mmol}$ ), $\mathrm{Cs}_{2} \mathrm{CO}_{3}$ (391 mg, $1.2 \mathrm{mmol}$ ), 2-chloro-5-iodopyridine (239 mg, $1.00 \mathrm{mmol}$ ), and $n$ hexanol $(249 \mu \mathrm{L}, 2.00 \mathrm{~mol})$ with toluene $(0.50 \mathrm{~mL})$ as solvent for $15 \mathrm{~h}$ at $80{ }^{\circ} \mathrm{C}$. Chromatographic purification (hexane / ethyl acetate 1:0 $\rightarrow$ 9:1) afforded the title compound as a colorless oil (179 mg, 84\%). ${ }^{1} \mathrm{H}$ NMR $\left(300 \mathrm{MHz}, \mathrm{CDCl}_{3}\right) \delta 8.03-8.02(1 \mathrm{H}, \mathrm{dd}, J=0.8,2.9 \mathrm{~Hz})$, 7.22-7.19 $(1 \mathrm{H}, \mathrm{dd}, J=0.8,8.7 \mathrm{~Hz}), 7.17-7.14(1 \mathrm{H}, \mathrm{dd}, J=2.9,8.7 \mathrm{~Hz}), 3.99-3.94(2 \mathrm{H}, \mathrm{t}, J=$ 
6.4), $1.80-1.73(2 \mathrm{H}, \mathrm{m}), 1.47-1.30(6 \mathrm{H}, \mathrm{m}), 0.92-0.87(3 \mathrm{H}, \mathrm{m}) .{ }^{13} \mathrm{C} \mathrm{NMR}\left(125 \mathrm{MHz}, \mathrm{CDCl}_{3}\right) \delta$ 154.9, 142.6, 137.0, 125.2, 124.7, 69.3, 31.9, 29.4, 26.0, 23.0, 14.4. IR ( $\mathrm{KBr}$ disc, $\left.\mathrm{cm}^{-1}\right) 2902$, 2863, 1516, 1454, 1245, 1016, 917, 814, 736, 697, 517. Anal. Calc. for $\mathrm{C}_{11} \mathrm{H}_{16} \mathrm{ClNO}$ : C 61.82, $\mathrm{H}$ 7.55. Found: C 62.02, H 7.65.

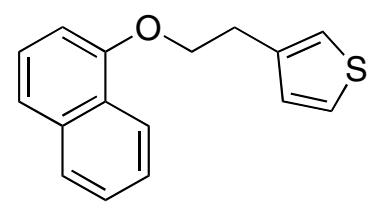

3-(2-(naphthalen-1-yloxy)ethyl)thiophene (Entry 16)

The general procedure was followed using CuI $(9.5 \mathrm{mg}, 0.050 \mathrm{mmol}), \mathrm{Me}_{4} \mathrm{Phen}(24 \mathrm{mg}, 0.10$

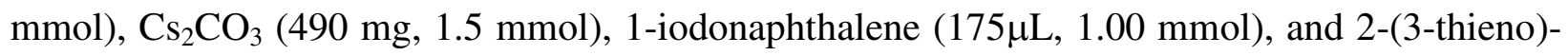
ethanol $(168 \mu \mathrm{L}, 1.50 \mathrm{~mol})$ with toluene $(0.50 \mathrm{~mL})$ as solvent for $24 \mathrm{~h} \mathrm{at} 110{ }^{\circ} \mathrm{C}$. Chromatographic purification (hexane / ethyl acetate 1:0 $\rightarrow$ 9:1) afforded the title compound as a tan oil (252 mg, 99\%). ${ }^{1} \mathrm{H}$ NMR (500 MHz, $\left.\mathrm{CDCl}_{3}\right) \delta 8.31-8.29(1 \mathrm{H}, \mathrm{m}), 7.82(1 \mathrm{H}, \mathrm{dd}, J=2.1$, $6.4 \mathrm{~Hz}), 7.53-7.48(1 \mathrm{H}, \mathrm{m}), 7.45(1 \mathrm{H}, \mathrm{d}, J=8.2 \mathrm{~Hz}), 7.39(1 \mathrm{H}, \mathrm{d}, J=8.0 \mathrm{~Hz}), 7.31(1 \mathrm{H}, \mathrm{dd}, J=$ 2.9, $4.9 \mathrm{~Hz}), 7.19-7.18(1 \mathrm{H}, \mathrm{m}), 7.14(1 \mathrm{H}, \mathrm{dd}, J=1.2,4.9 \mathrm{~Hz}), 6.83(1 \mathrm{H}, \mathrm{d}, J=7.5 \mathrm{~Hz}), 4.38$ $(2 \mathrm{H}, \mathrm{d}, J=6.7 \mathrm{~Hz}), 3.31(2 \mathrm{H}, \mathrm{d}, J=6.4 \mathrm{~Hz}) .{ }^{13} \mathrm{C} \mathrm{NMR}\left(125 \mathrm{MHz}, \mathrm{CDCl}_{3}\right) \delta$ 154.7. 138.9. 134.7. 128.7. 127.7. 126.6. 126.0. 125.9. 125.7. 125.4. 122.2. 121.8. 120.5. 104.8. 68.4. 30.5. IR $\left(\mathrm{KBr}\right.$ disc, $\left.\mathrm{cm}^{-1}\right)$ 3052, 2928, 2873, 1594, 1580, 1508, 1460, 1405, 1269, 1240, 1100, 1071, 1020, 790. Anal. for $\mathrm{C}_{16} \mathrm{H}_{14} \mathrm{OS}$ : C 75.55, H 5.55. Found: C 75.28, H 5.50.<smiles>N#Cc1cccc(OCCc2cccnc2)c1</smiles>

3-(2-(pyridin-3-yl)ethoxy)benzonitrile (Entry 17)

The general procedure was followed using CuI $(9.5 \mathrm{mg}, 0.050 \mathrm{mmol}), \mathrm{Me}_{4} \mathrm{Phen}(24 \mathrm{mg}, 0.10$ $\mathrm{mmol}), \mathrm{Cs}_{2} \mathrm{CO}_{3}(490 \mathrm{mg}, 1.5 \mathrm{mmol}), 3$-iodobenzonitrile $(229 \mathrm{mg}, 1.00 \mathrm{mmol})$, and 2-(3- 
pyridyl)ethanol $(172 \mu \mathrm{L}, 1.50 \mathrm{~mol})$ with toluene $(0.50 \mathrm{~mL})$ as solvent for $24 \mathrm{~h}$ at $80{ }^{\circ} \mathrm{C}$. Chromatographic purification (hexane / ethyl acetate 1:0 $\rightarrow$ 3:1) afforded the title compound as a clear oil $(174 \mathrm{mg}, 78 \%) .{ }^{1} \mathrm{H}$ NMR $\left(500 \mathrm{MHz}, \mathrm{CDCl}_{3}\right) \delta 8.56(1 \mathrm{H}, \mathrm{d}, J=2.2 \mathrm{~Hz}), 8.22(1 \mathrm{H}, \mathrm{dd}, J$ $=1.6,4.7 \mathrm{~Hz}), 7.65-7.60(1 \mathrm{H}, \mathrm{m}), 7.40-7.33(1 \mathrm{H}, \mathrm{m}), 7.29-7.23(2 \mathrm{H}, \mathrm{m}), 7.13-7.09(2 \mathrm{H}, \mathrm{m}), 4.20$ $(2 \mathrm{H}, \mathrm{t}, J=6.5 \mathrm{~Hz}), 3.12(2 \mathrm{H}, \mathrm{t}, J=6.5 \mathrm{~Hz}) .{ }^{13} \mathrm{C} \mathrm{NMR}\left(125 \mathrm{MHz}, \mathrm{CDCl}_{3}\right) \delta 158.8,150.5,148.5$, 136.6, 133.6, 130.6, 125.0, 123.6, 119.9, 118.8, 117.6, 113.4, 68.5, 33.0. IR ( $\mathrm{KBr}$ disc, $\left.\mathrm{cm}^{-1}\right)$ 3033, 2934, 2878, 2230, 1597, 1578, 1480, 1431, 1328, 1292, 1148, 1033, 971, 715, 682. Anal. Calc. for $\mathrm{C}_{14} \mathrm{H}_{12} \mathrm{~N}_{2} \mathrm{O}$ : C 74.98, H 5.39.<smiles>c1ccc(OC2CCCCC2)nc1</smiles>

2-(cyclohexyloxy)pyridine (Entry 18)

The general procedure was followed using $\mathrm{CuI}(9.5 \mathrm{mg}, 0.050 \mathrm{mmol}), \mathrm{Me}_{4} \mathrm{Phen}(24 \mathrm{mg}, 0.10$ mmol), $\mathrm{Cs}_{2} \mathrm{CO}_{3}(490 \mathrm{mg}, 1.5 \mathrm{mmol}), 4 \AA$ molecular sieves $(200 \mathrm{mg}$, flame activated under vacuum) 2-iodopyridine (106 $\mu \mathrm{L}, 1.00 \mathrm{mmol})$, and cyclohexanol $(158 \mu \mathrm{L}, 1.50 \mathrm{mmol})$ with toluene $(0.50 \mathrm{~mL})$ as solvent for $24 \mathrm{~h}$ at $110{ }^{\circ} \mathrm{C}$. Chromatographic purification (hexane / ethyl acetate 1:0 $\rightarrow$ 9:1) afforded the title compound as a clear oil (164 mg, 93\%). ${ }^{1} \mathrm{H}$ NMR (500 $\left.\mathrm{MHz}, \mathrm{CDCl}_{3}\right) \delta 8.14(1 \mathrm{H}, \mathrm{ddd}, J=0.8,2.1,5.0 \mathrm{~Hz}), 7.54(1 \mathrm{H}, \mathrm{ddd}, J=2.0,7.2,8.4 \mathrm{~Hz}), 6.81$ $(1 \mathrm{H}, \mathrm{ddd}, J=0.9,5.0,7.1 \mathrm{~Hz}), 6.69(1 \mathrm{H}, \mathrm{dt}, J=8.4,0.8 \mathrm{~Hz}), 5.06-5.00(1 \mathrm{H}, \mathrm{m}), 2.05-2.01(2 \mathrm{H}$, m), $1.82-1.76(2 \mathrm{H}, \mathrm{m}), 1.66-1.39(4 \mathrm{H}, \mathrm{m}), 1.34-1.25(2 \mathrm{H}, \mathrm{m}) .{ }^{13} \mathrm{C}$ NMR $\left(125 \mathrm{MHz}, \mathrm{CDCl}_{3}\right) \delta$ 163.7, 147.1, 138.7, 116.4, 111.9, 73.2, 32.1, 25.9, 24.2. IR (KBr disc, $\left.\mathrm{cm}^{-1}\right)$ 3025, 2942, 1614 1516, 1328, 1249, 1161, 1110, 1061, 961, 837, 749. Anal. Calc. for $\mathrm{C}_{11} \mathrm{H}_{15} \mathrm{NO}$ : C 74.54, H 8.53. Found: C 74.50, H 8.63 . 


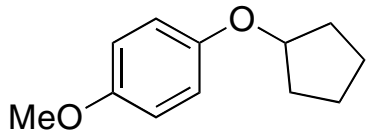

1-(cyclopentyloxy)-4-methoxybenzene (Entry 19) $)^{\text {viii }}$

The general procedure was followed using $\mathrm{CuI}(9.5 \mathrm{mg}, 0.050 \mathrm{mmol}), \mathrm{Me}_{4} \mathrm{Phen}(24 \mathrm{mg}, 0.10$ mmol), $\mathrm{Cs}_{2} \mathrm{CO}_{3}$ (490 mg, $1.5 \mathrm{mmol}$ ), 4-iodoanisole (234 mg, $1.00 \mathrm{mmol}$ ), and cyclopentanol $(136 \mu \mathrm{L}, 1.50 \mathrm{~mol})$ with toluene $(0.50 \mathrm{~mL})$ as solvent for $24 \mathrm{~h}$ at $110{ }^{\circ} \mathrm{C}$. Chromatographic purification (hexane / ethyl acetate 1:0 $\rightarrow$ 9:1) afforded the title compound as a clear oil (168 mg mg, 88\%). ${ }^{1} \mathrm{H}$ NMR $\left(500 \mathrm{MHz}, \mathrm{CDCl}_{3}\right) \delta 6.83(4 \mathrm{H}, \mathrm{s}), 4.71-4.66(1 \mathrm{H}, \mathrm{m}), 3.78(3 \mathrm{H}, \mathrm{s}), 1.90-$ $1.76(6 \mathrm{H}, \mathrm{m}), 1.65-1.56(2 \mathrm{H}, \mathrm{m}) .{ }^{13} \mathrm{C} \mathrm{NMR}\left(125 \mathrm{MHz}, \mathrm{CDCl}_{3}\right) \delta 152.3,116.8,114.8,80.0,55.9$, 33.0, 24.2. IR ( $\mathrm{KBr}$ disc, $\left.\mathrm{cm}^{-1}\right)$ 2960, 2872, 1833, 1507, 1465, 1441, 1231, 1173, 1106, 1040, 990, 824. Anal. Calc. for $\mathrm{C}_{12} \mathrm{H}_{16} \mathrm{O}_{2}$ : C 74.97, H 8.39. Found: C 74.56, H 8.25.<smiles>FC(F)(F)c1ccc(OC2CCCc3ccccc32)cc1</smiles>

1-(4-(trifluoromethyl)phenoxy)-1,2,3,4-tetrahydronaphthalene (Entry 20)

The general procedure was followed using $\mathrm{CuI}(9.5 \mathrm{mg}, 0.050 \mathrm{mmol}), \mathrm{Me}_{4} \mathrm{Phen}(24 \mathrm{mg}, 0.10$ mmol), $\mathrm{Cs}_{2} \mathrm{CO}_{3}(490 \mathrm{mg}, 1.5 \mathrm{mmol})$, 4-iodobenzotrifluoride (147 $\left.\mu \mathrm{L}, 1.00 \mathrm{mmol}\right)$, and ( \pm )-1tetralol (222 mg, $1.50 \mathrm{~mol})$ with toluene $(0.50 \mathrm{~mL})$ as solvent for $24 \mathrm{~h}$ at $110{ }^{\circ} \mathrm{C}$. Chromatographic purification (hexane / ethyl acetate 1:0 $\rightarrow$ 9:1) afforded the title compound as a clear oil (217 mg, 75\%). ${ }^{1} \mathrm{H}$ NMR $\left(500 \mathrm{MHz}^{\mathrm{CDCl}}{ }_{3}\right) \delta 7.59(2 \mathrm{H}, \mathrm{d}, J=8.8 \mathrm{~Hz}), 7.36-7.19(4 \mathrm{H}$, m), $7.10(\mathrm{~d}, J=8.8 \mathrm{~Hz}), 5.47(1 \mathrm{H}, \mathrm{t}, J=4.3 \mathrm{~Hz}), 2.96-2.91(1 \mathrm{H}, \mathrm{m}), 2.84-2.78(1 \mathrm{H}, \mathrm{m}), 2.21-$ $2.15(1 \mathrm{H}, \mathrm{m}), 2.09-1.99(2 \mathrm{H}, \mathrm{m}), 1.87-1.80(1 \mathrm{H}, \mathrm{m}) .{ }^{13} \mathrm{C} \mathrm{NMR}\left(125 \mathrm{MHz}, \mathrm{CDCl}_{3}\right) \delta 138.0$, $135.1,129.6,129.4,128.4,127.3,127.2,127.1,126.4,116.1,74.2,29.2,28.0,18.9 . \mathrm{IR}(\mathrm{KBr}$ 
disc, $\mathrm{cm}^{-1}$ ) 3024, 2942, 1614, 1516, 1328, 1250, 1161, 1110, 1061, 961, 837, 749. Anal. Calc. for $\mathrm{C}_{17} \mathrm{H}_{15} \mathrm{~F}_{3} \mathrm{OX}$ : C 69.85, H 5.17. Found: C 69.74, H 5.24.<smiles>Cc1ccc(OCCCCC(C)O)cc1</smiles>

1-(p-tolyloxy)hexan-5-ol (Entry 21)

The general procedure was followed using $\mathrm{CuI}(9.5 \mathrm{mg}, 0.050 \mathrm{mmol}), \mathrm{Me}_{4}$ Phen $(24 \mathrm{mg}, 0.10$ mmol), $\mathrm{Cs}_{2} \mathrm{CO}_{3}$ (490 mg, $1.5 \mathrm{mmol}$ ), 4-iodotoluene (218 mg, $1.00 \mathrm{mmol}$ ), and 1,5-hexanediol $(181 \mu \mathrm{L}, 1.50 \mathrm{~mol})$ with toluene $(0.50 \mathrm{~mL})$ as solvent for $24 \mathrm{~h}$ at $80{ }^{\circ} \mathrm{C}$. Chromatographic purification (hexane / ethyl acetate 1:0 $\rightarrow$ 4:1) afforded the title compound as a clear oil (156 mg, 75\%). ${ }^{1} \mathrm{H}$ NMR (500 MHz, $\left.\mathrm{CDCl}_{3}\right) \delta$ 7.09-7.07 (2H, m), 6.82-6.79 (2H, m), $3.95(2 \mathrm{H}, \mathrm{t}, J=$ $6.5 \mathrm{~Hz}), 6.87-3.81(1 \mathrm{H}, \mathrm{m}), 2.30(3 \mathrm{H}, \mathrm{t}), 1.83-1.78(2 \mathrm{H}, \mathrm{m}), 1.61-1.47(6 \mathrm{H}, \mathrm{m}), 1.22(3 \mathrm{H}, \mathrm{dd}, J=$ 0.6, 6.3 Hz). ${ }^{13} \mathrm{C}$ NMR (125 MHz, $\left.\mathrm{CDCl}_{3}\right) \delta 157.1,130.0,129.9,114.5,68.2,68.0,39.2,29.5$, 23.7, 22.6, 20.7. IR (KBr disc, $\left.\mathrm{cm}^{-1}\right) 3363$ (br), 3031, 2940, 2867, 1614, 1584, 1512, 1474, 1376, 1291, 1244, 1176, 1111, 1037, 952, 818, 511.

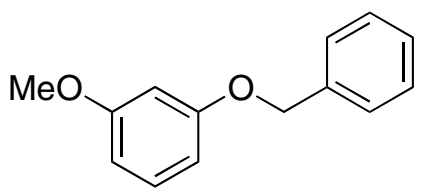

1-(benzyloxy)-3-methoxybenzene (Entry 22) ${ }^{\mathrm{ix}}$

The general procedure was followed using CuI (19 mg, $0.050 \mathrm{mmol}), \mathrm{Me}_{4} \mathrm{Phen}(48 \mathrm{mg}, 0.10$ mmol), $\mathrm{Cs}_{2} \mathrm{CO}_{3}$ (490 mg, $\left.1.5 \mathrm{mmol}\right)$, 3-bromoanisole (127 $\left.\mu \mathrm{L}, 1.00 \mathrm{mmol}\right)$, and benzyl alcohol $(155 \mu \mathrm{L}, 1.50 \mathrm{~mol})$ with toluene $(0.50 \mathrm{~mL})$ as solvent for $24 \mathrm{~h}$ at $110{ }^{\circ} \mathrm{C}$. Chromatographic purification (hexane / ethyl acetate 1:0 $\rightarrow$ 9:1) afforded the title compound as a clear oil (199 mg, 93\%). ${ }^{1} \mathrm{H}$ NMR (500 MHz, $\left.\mathrm{CDCl}_{3}\right) \delta$ 7.51-7.36 (5H, m), 7.23 (1H, m), 6.64-6.57 (3H, m), $5.08(2 \mathrm{H}, \mathrm{s}), 3.82(3 \mathrm{H}, \mathrm{s}) .{ }^{13} \mathrm{C} \mathrm{NMR}\left(125 \mathrm{MHz}, \mathrm{CDCl}_{3}\right) \delta 161.0,160.2,137.1,130.1,128.8$ 
128.1, 127.7, 107.1, 106.7, 101.5, 70.2, 55.4. IR (KBr disc, $\left.\mathrm{cm}^{-1}\right)$ 3032, 2939, 2835, 1592, 1492 , $1453,1381,1288,1264,1199,1151,1082,1045,835,761,734,697$. Anal. Calc. for $\mathrm{C}_{14} \mathrm{H}_{14} \mathrm{O}_{2}$ : C 78.48, H 6.59. Found: C 78.39, H 6.59.<smiles>CCCCCCOc1cccc(OC)c1</smiles>

3-(hexyloxy)-anisole (Entry 23) ${ }^{\mathrm{x}}$

The general procedure was followed using $\mathrm{CuI}(9.5 \mathrm{mg}, 0.050 \mathrm{mmol}), \mathrm{Me}_{4} \mathrm{Phen}(24 \mathrm{mg}, 0.10$ mmol), $\mathrm{Cs}_{2} \mathrm{CO}_{3}$ (490 mg, $\left.1.5 \mathrm{mmol}\right), 3$-bromoanisole (127 $\left.\mu \mathrm{L}, 1.00 \mathrm{mmol}\right)$, and $n$-hexanol (0.5 $\mathrm{mL}$ ) as solvent for $24 \mathrm{~h}$ at $130{ }^{\circ} \mathrm{C}$. Chromatographic purification (hexane / ethyl acetate 1:0 $\rightarrow$ 9:1) afforded the title compound as a clear oil $(160 \mathrm{mg}, 77 \%) .{ }^{1} \mathrm{H}$ NMR $\left(500 \mathrm{MHz}, \mathrm{CDCl}_{3}\right) \delta$ $7.19(1 \mathrm{H}, \mathrm{t}, J=4.5 \mathrm{~Hz}), 6.53-6.48(3 \mathrm{H}, \mathrm{m}), 3.95(2 \mathrm{H}, \mathrm{t}, J=6.7 \mathrm{~Hz}), 3.79(3 \mathrm{H}, \mathrm{s}), 1.81-1.76(2 \mathrm{H}$, m), 1.49-1.45 (2H, m), 1.38-1.33 (4H, m), 0.94-0.91 (3H, m). $\left.{ }^{13} \mathrm{C} \mathrm{NMR} \mathrm{(125} \mathrm{MHz,} \mathrm{CDCl}_{3}\right) \delta$ 161.0, 160.6, 130.0, 106.8, 106.3, 101.1, 77.0, 55.5, 31.8, 29.5, 26.0, 22.8, 14.3. IR (KBr disc, $\left.\mathrm{cm}^{-1}\right) 3000,2933,28871,1599,1493,1468,1455,1334,1287,1265,1201,1153,1046,835$, $762,687$. 


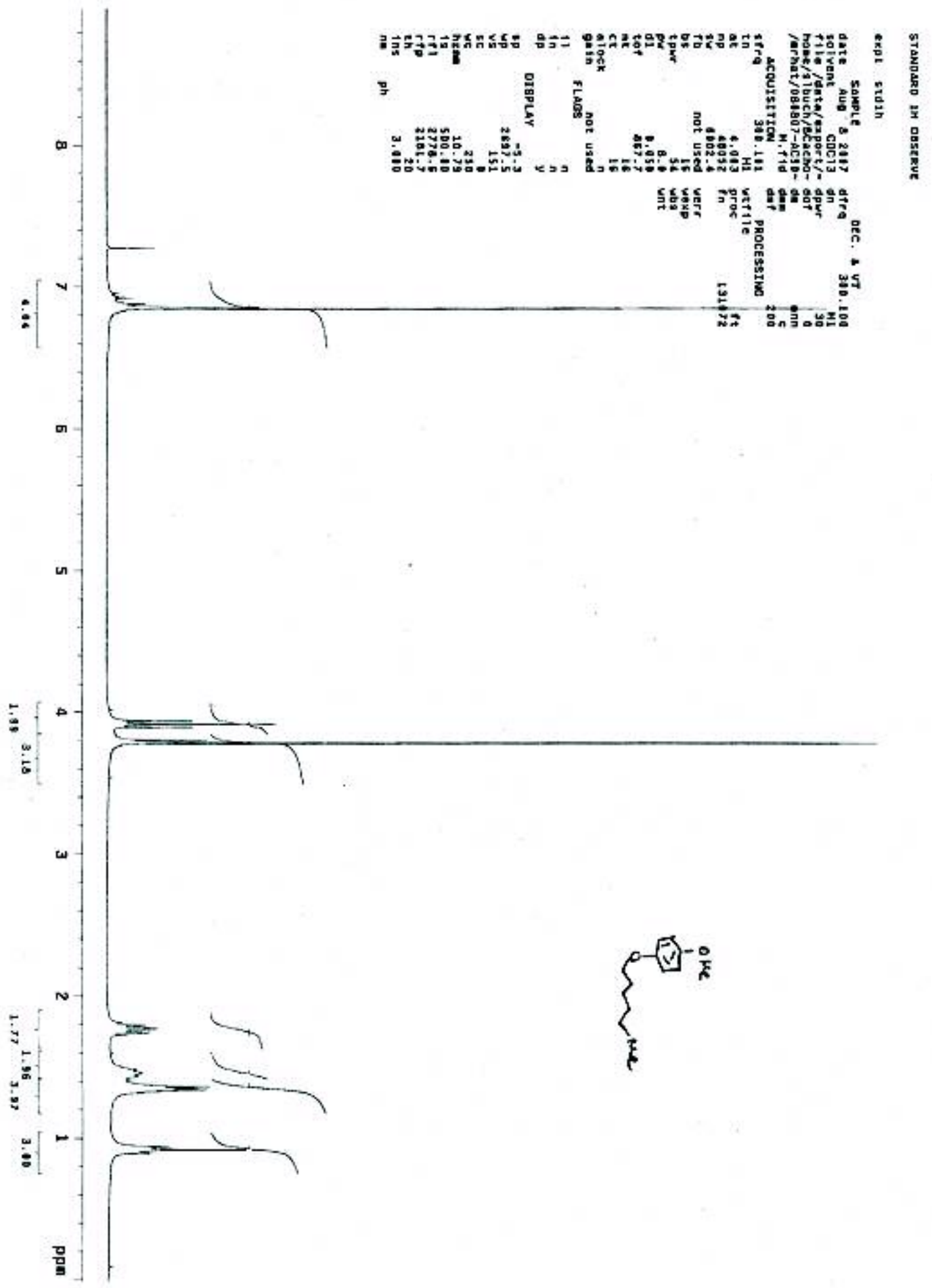




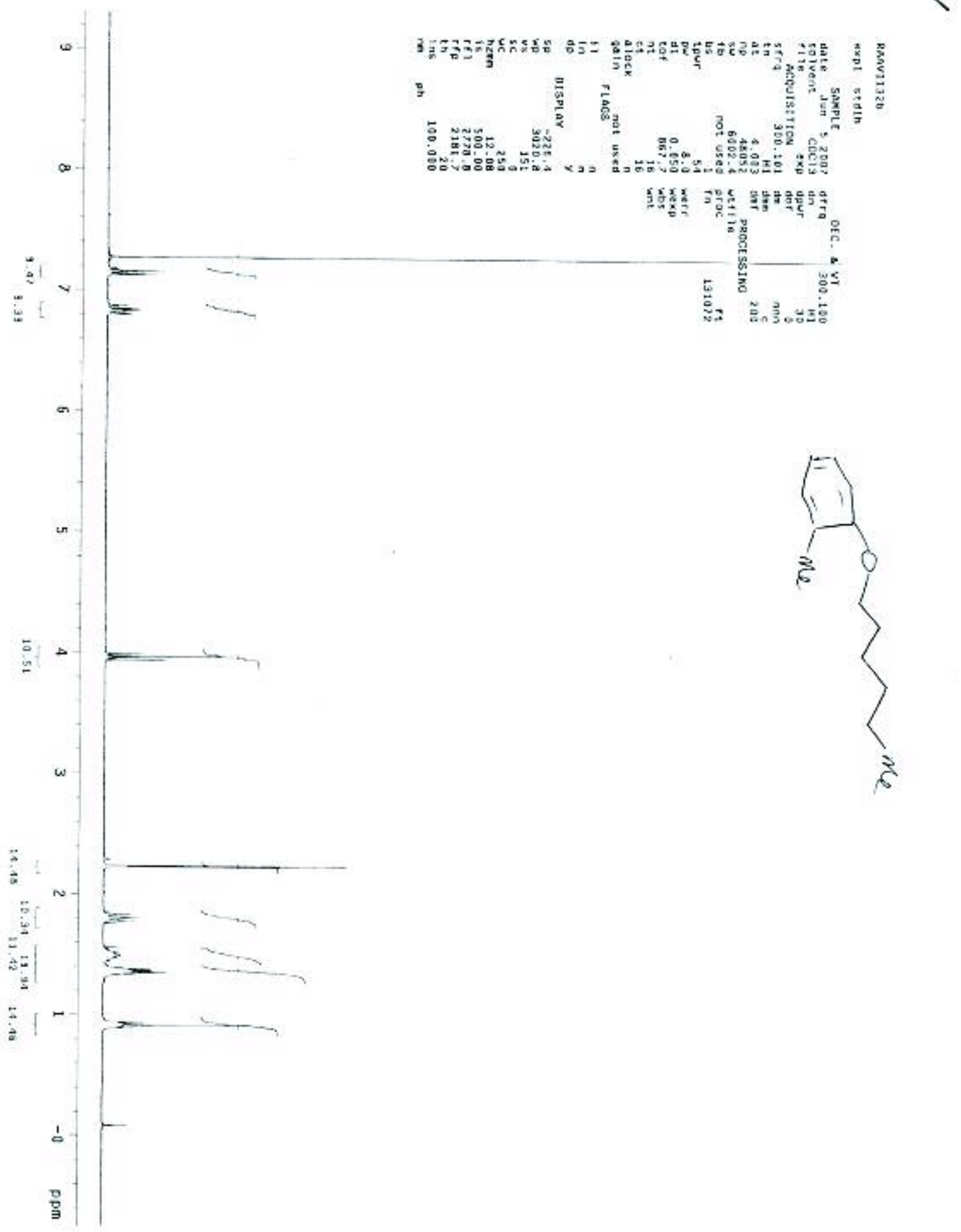




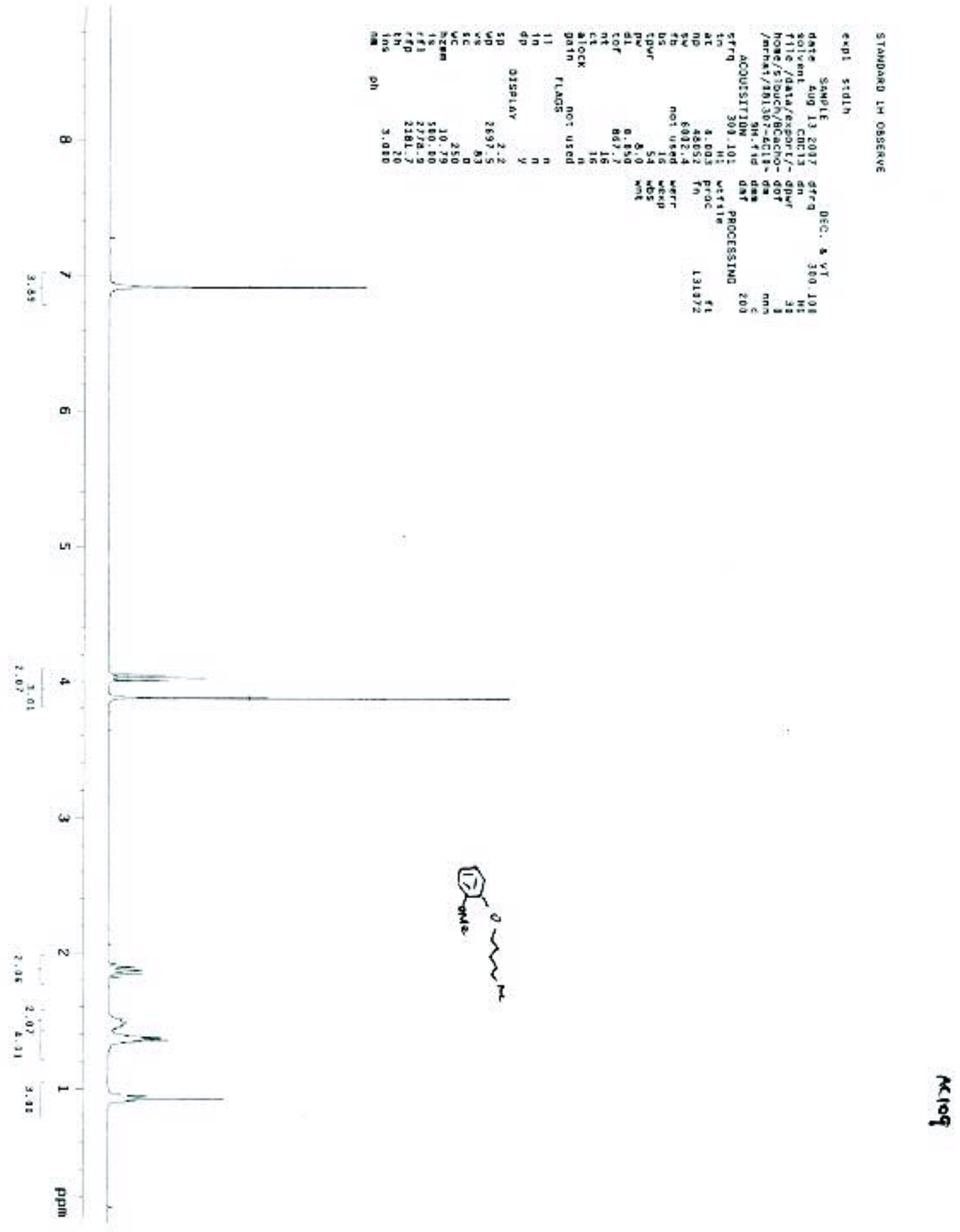




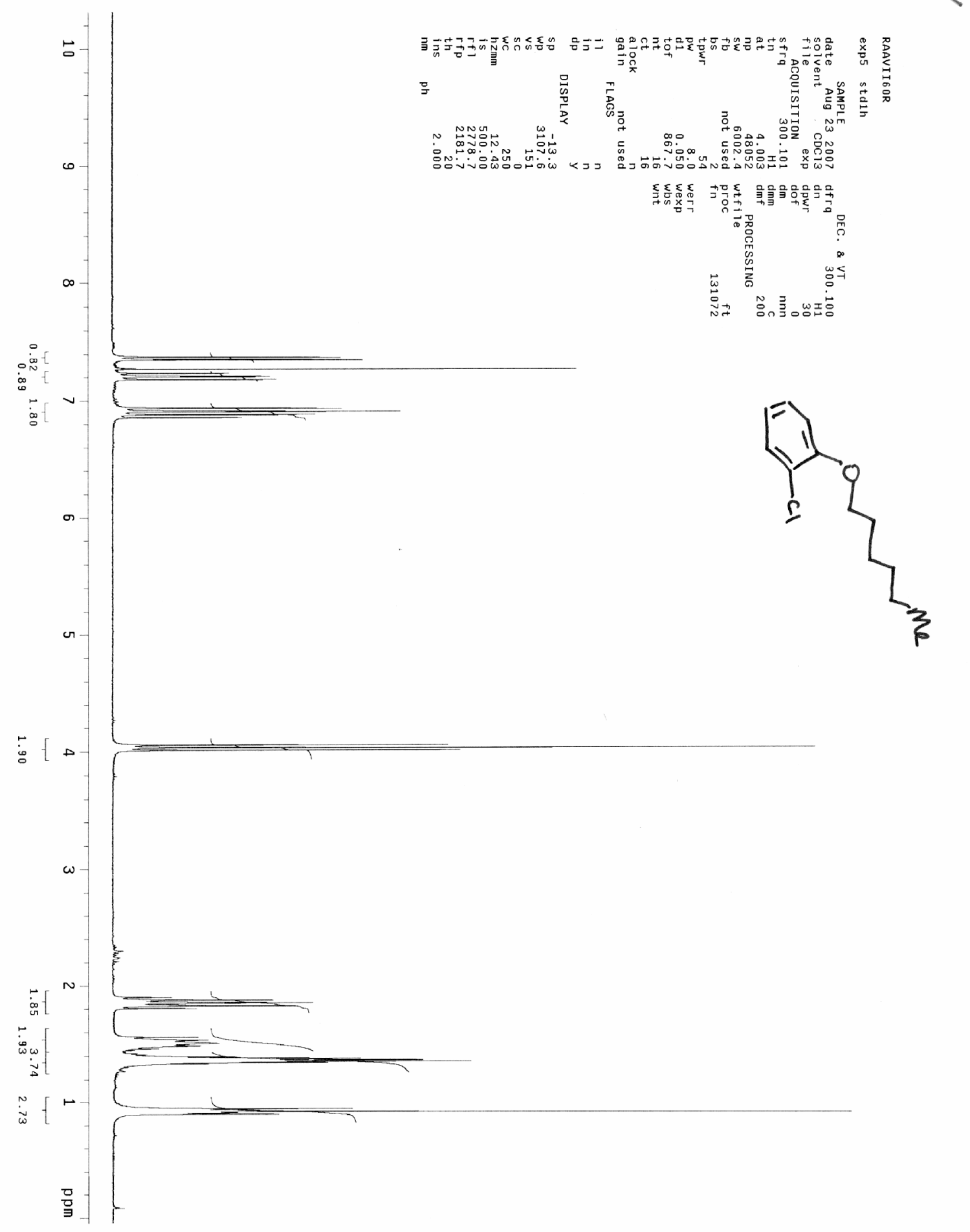




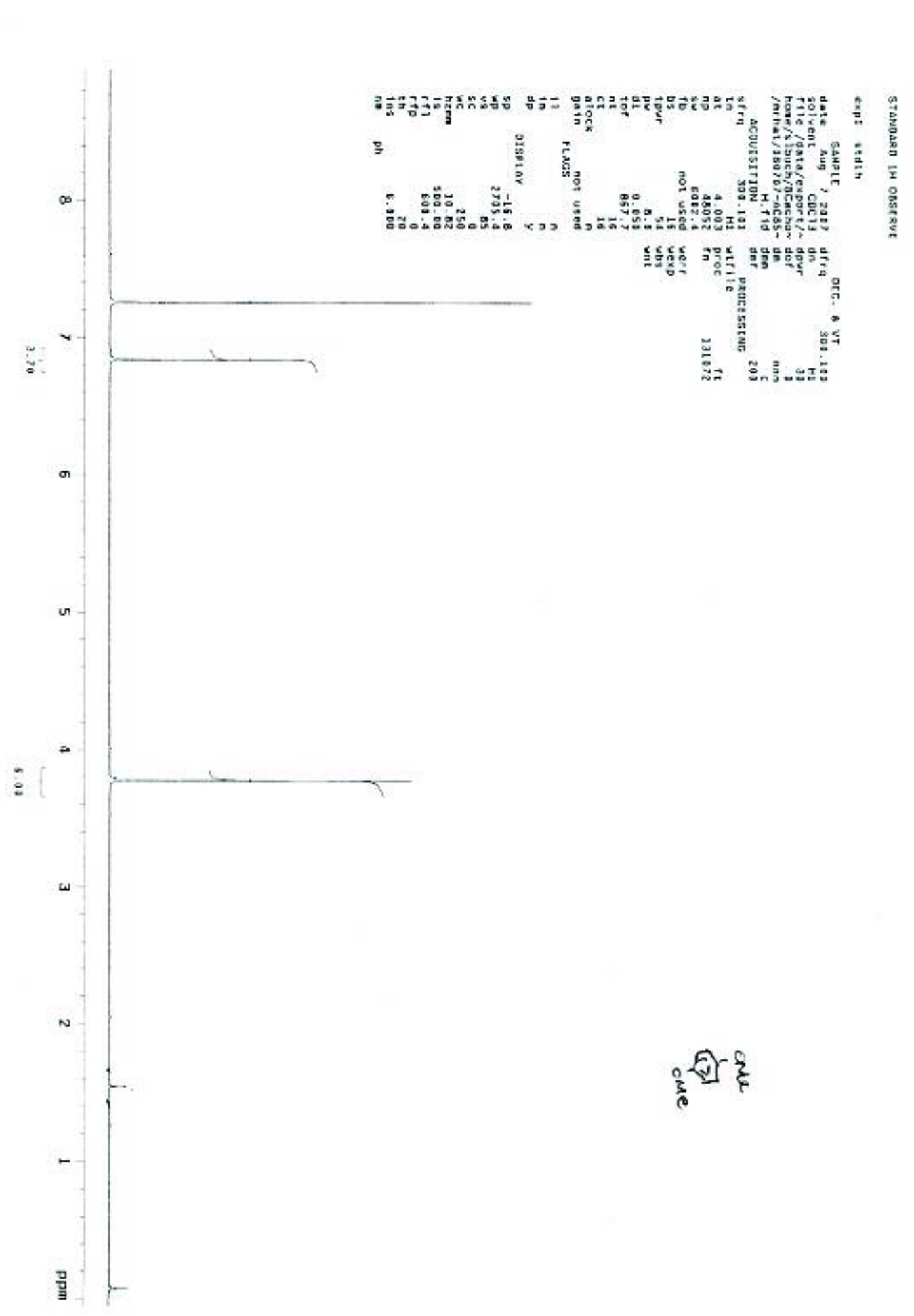




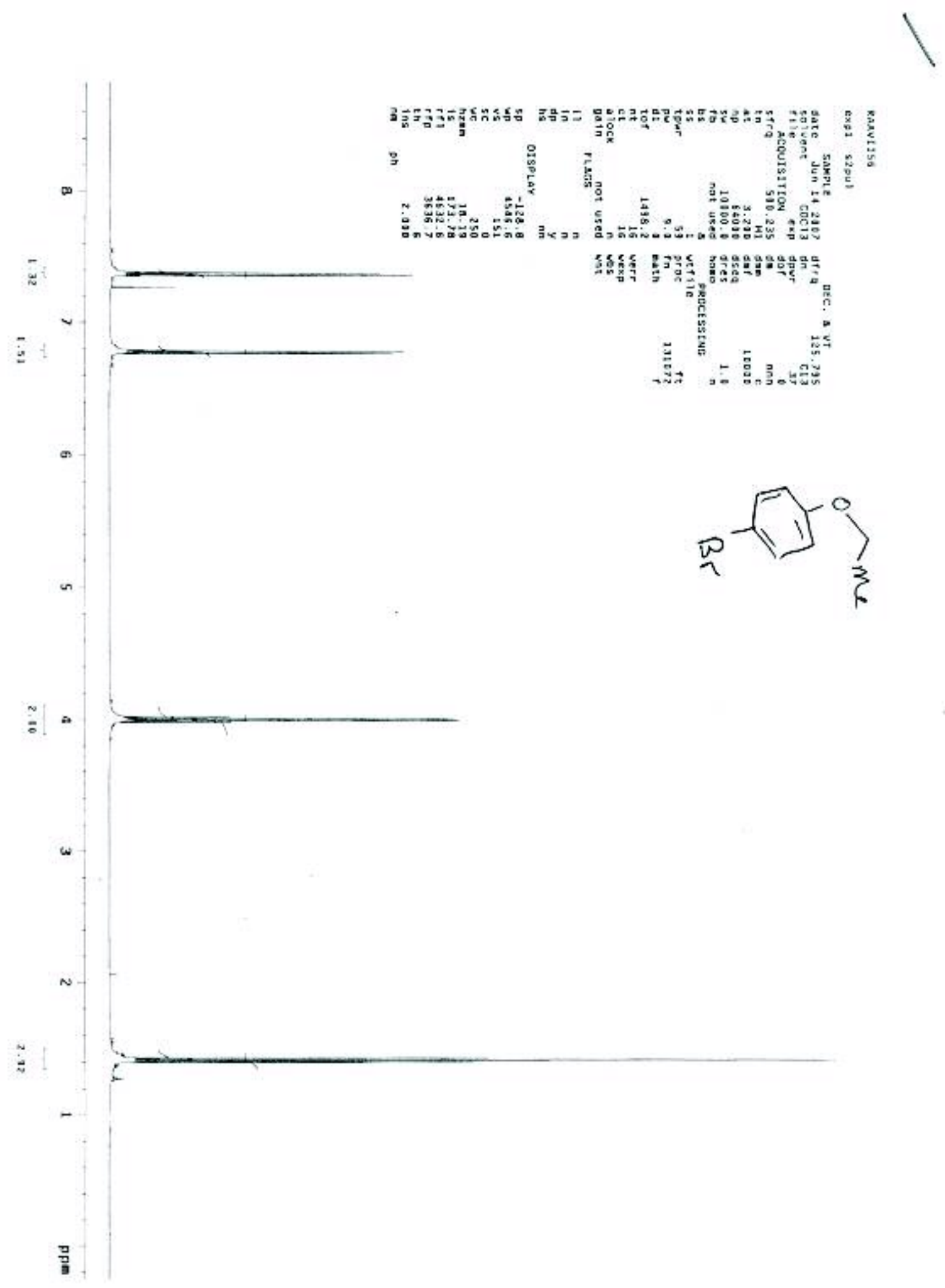




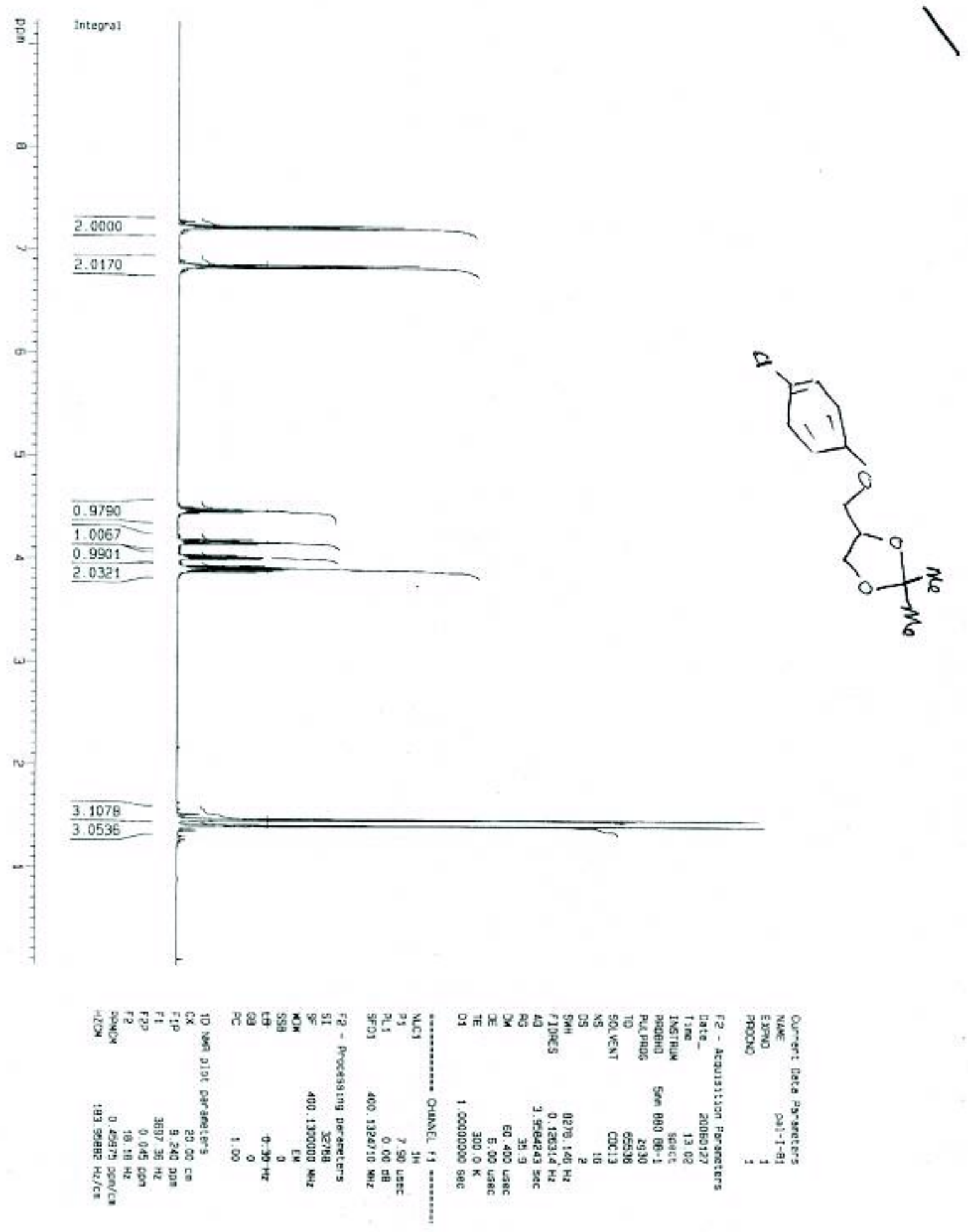




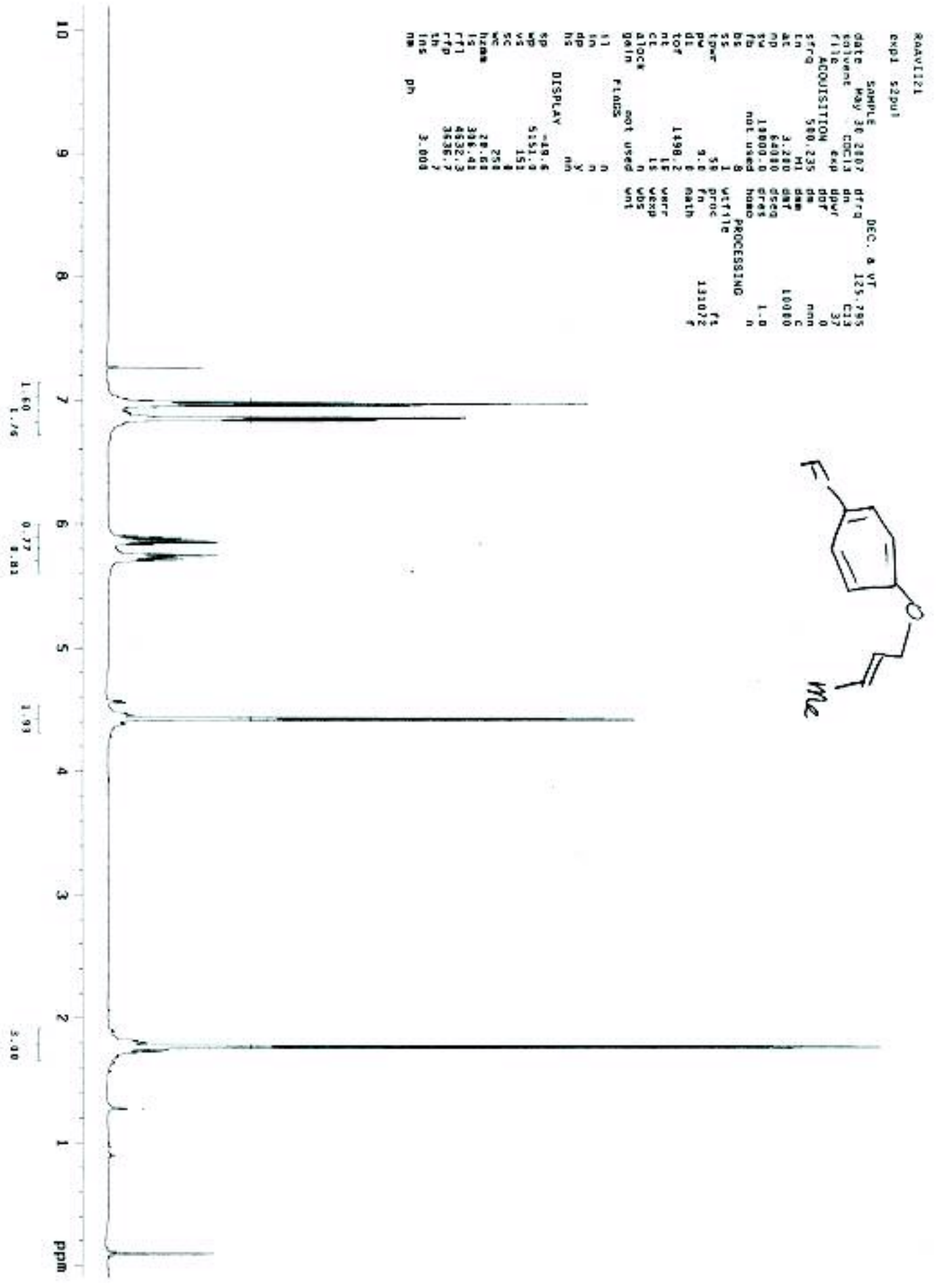




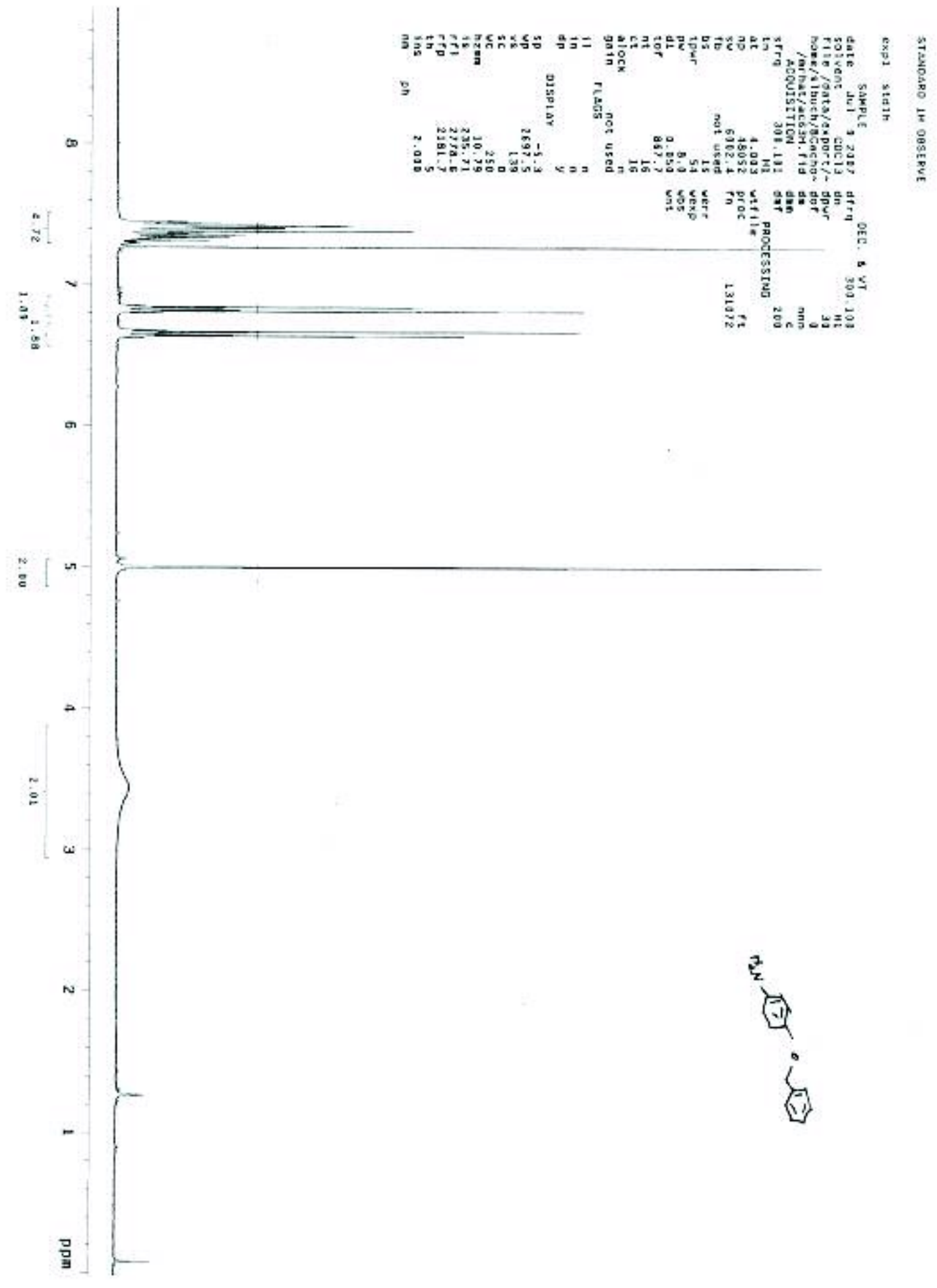




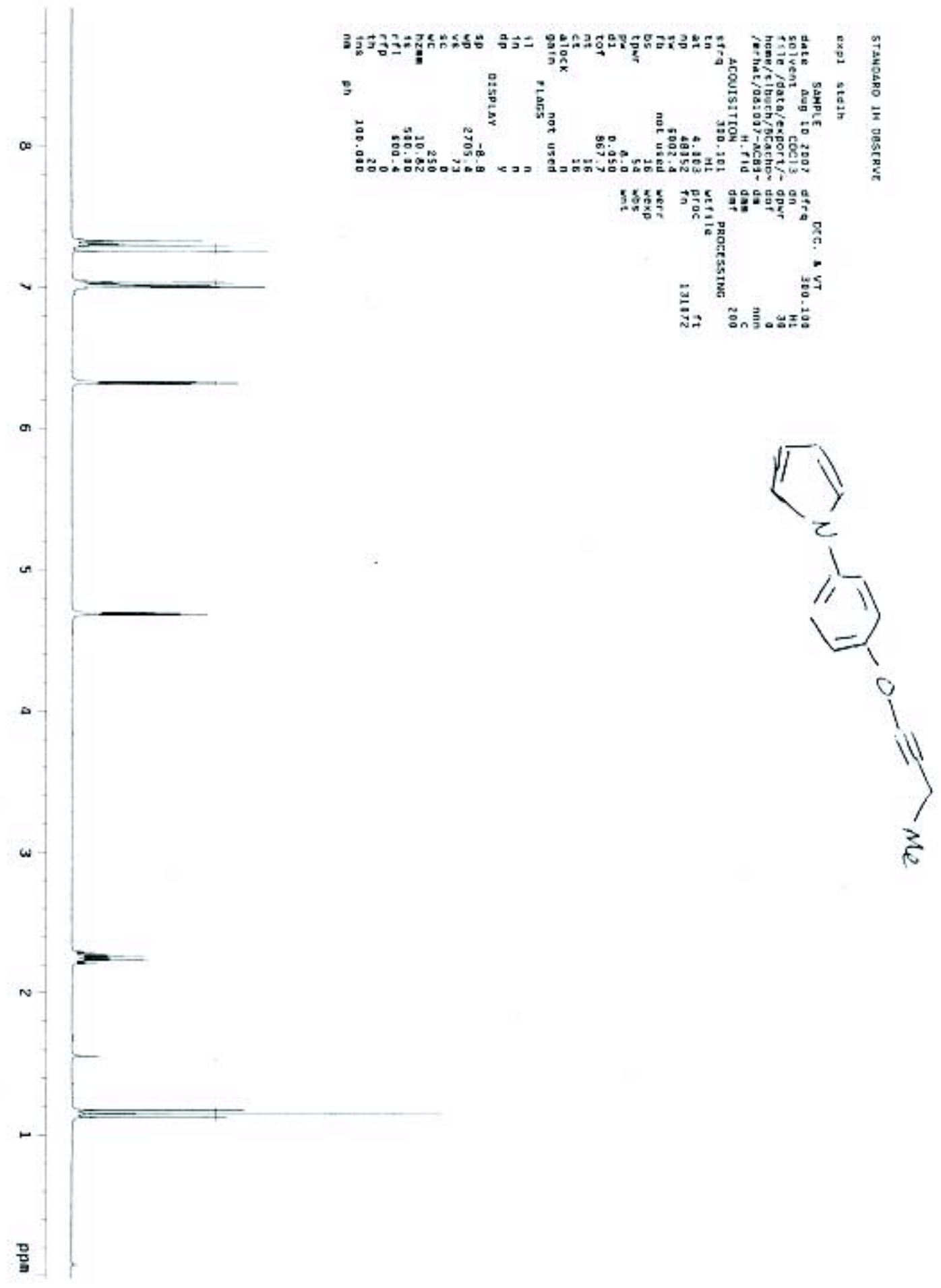




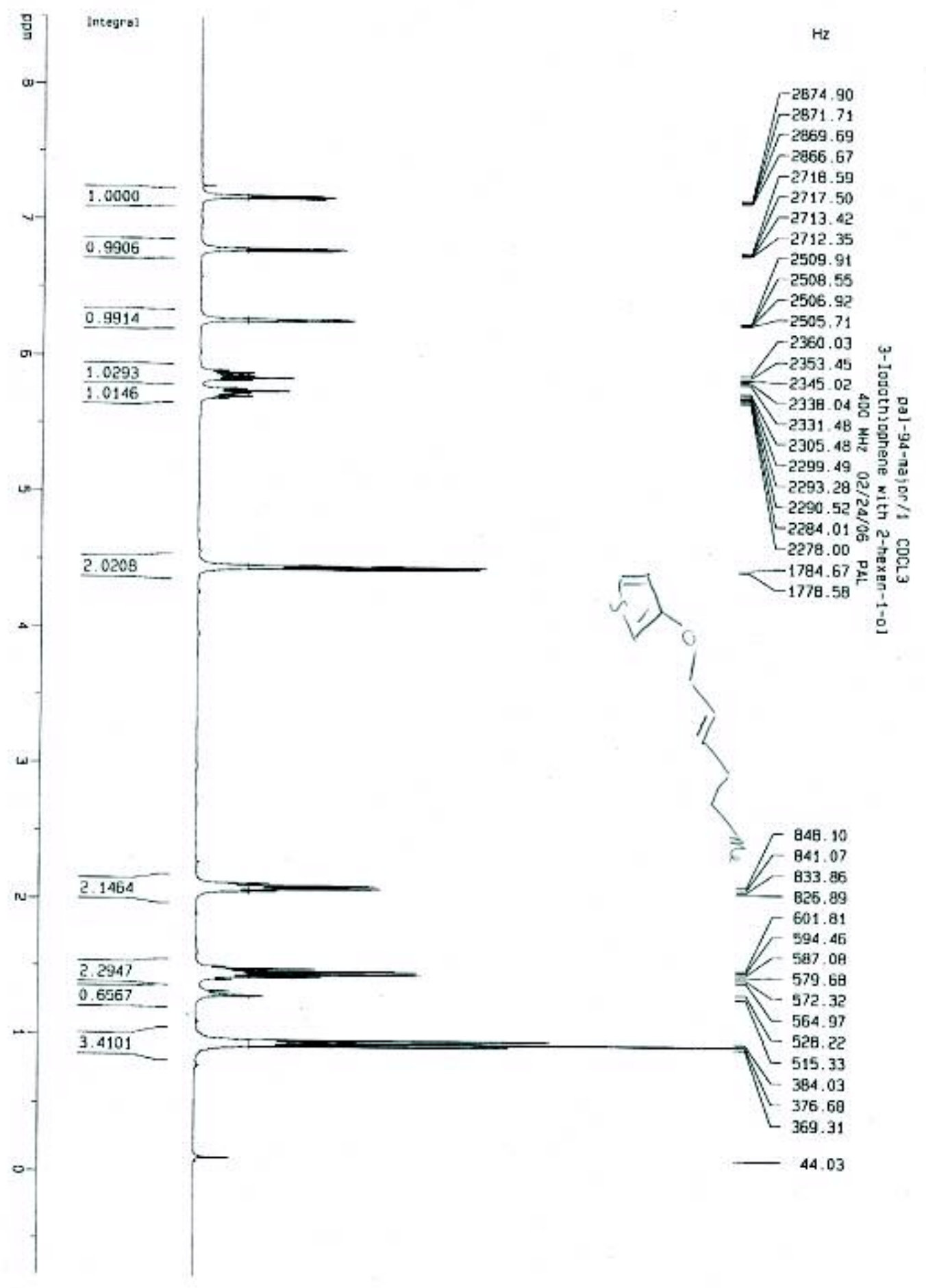




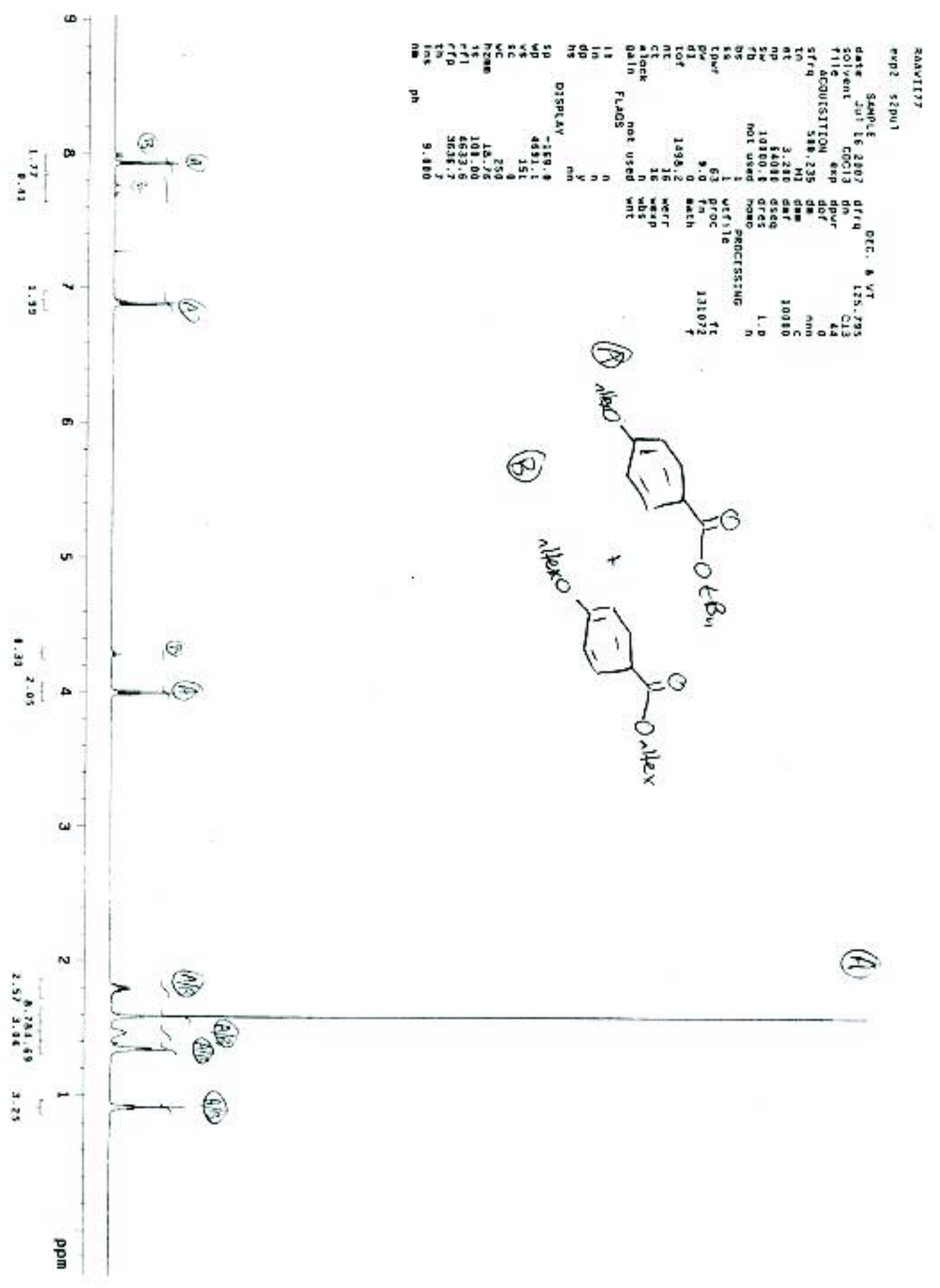




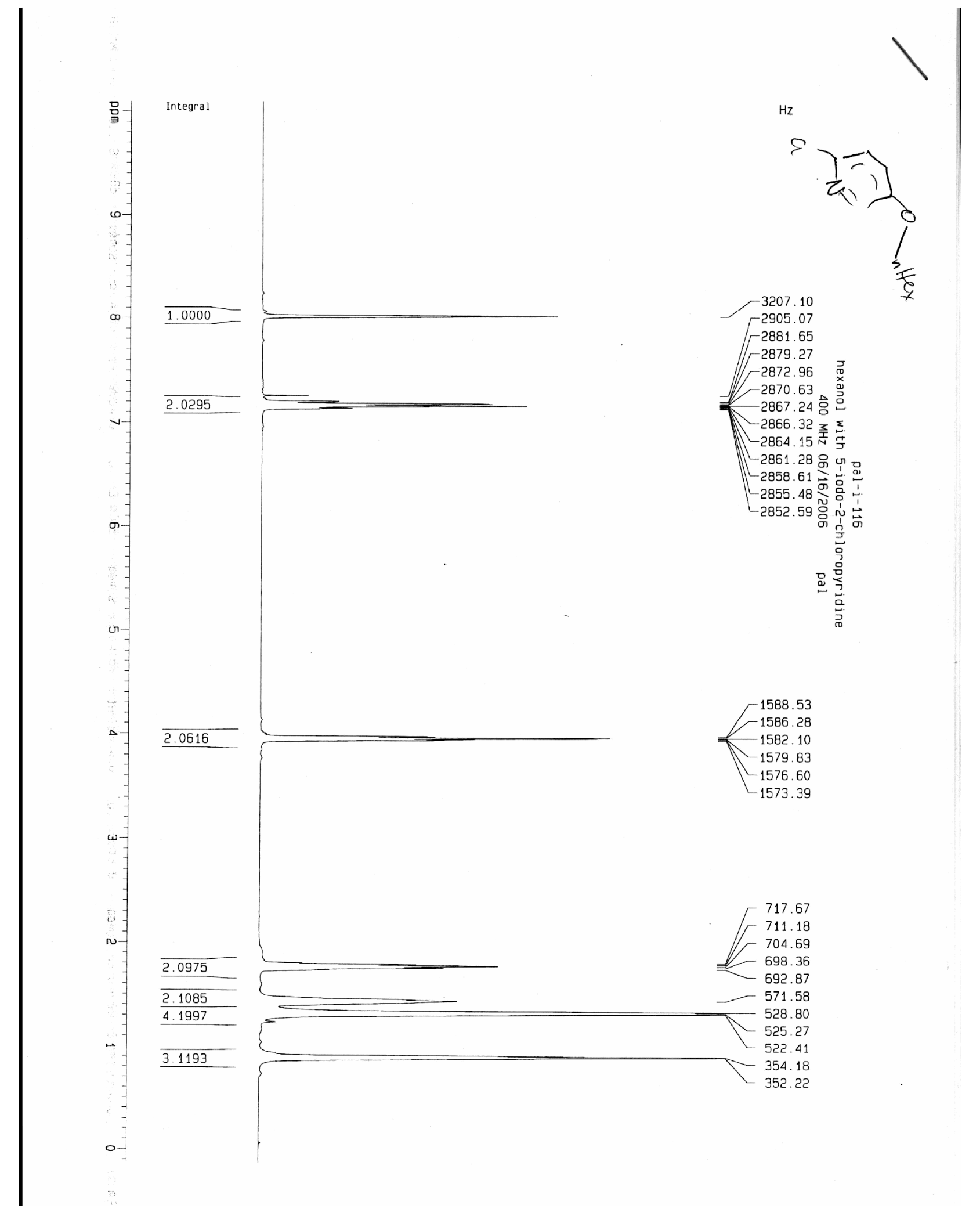




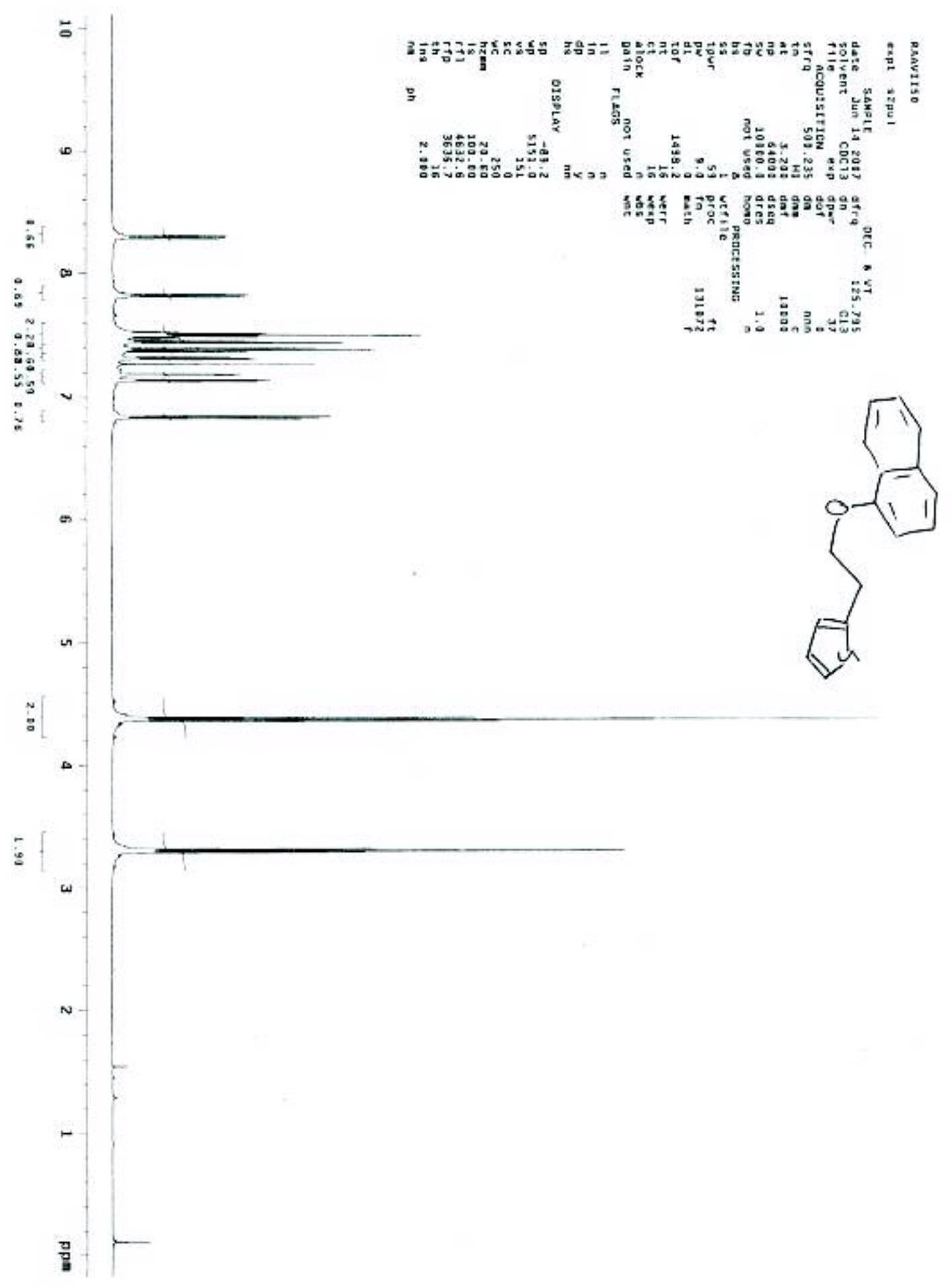




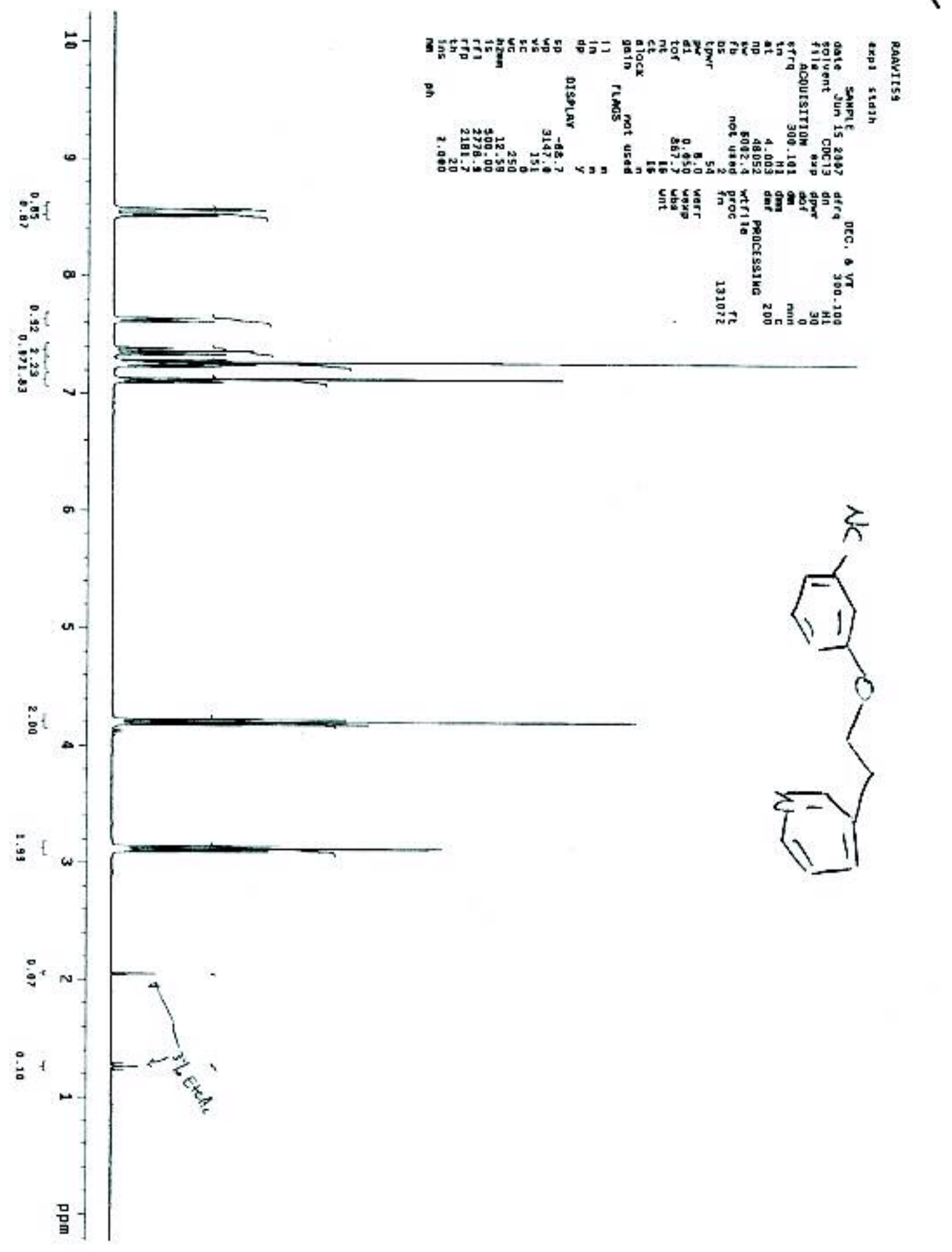




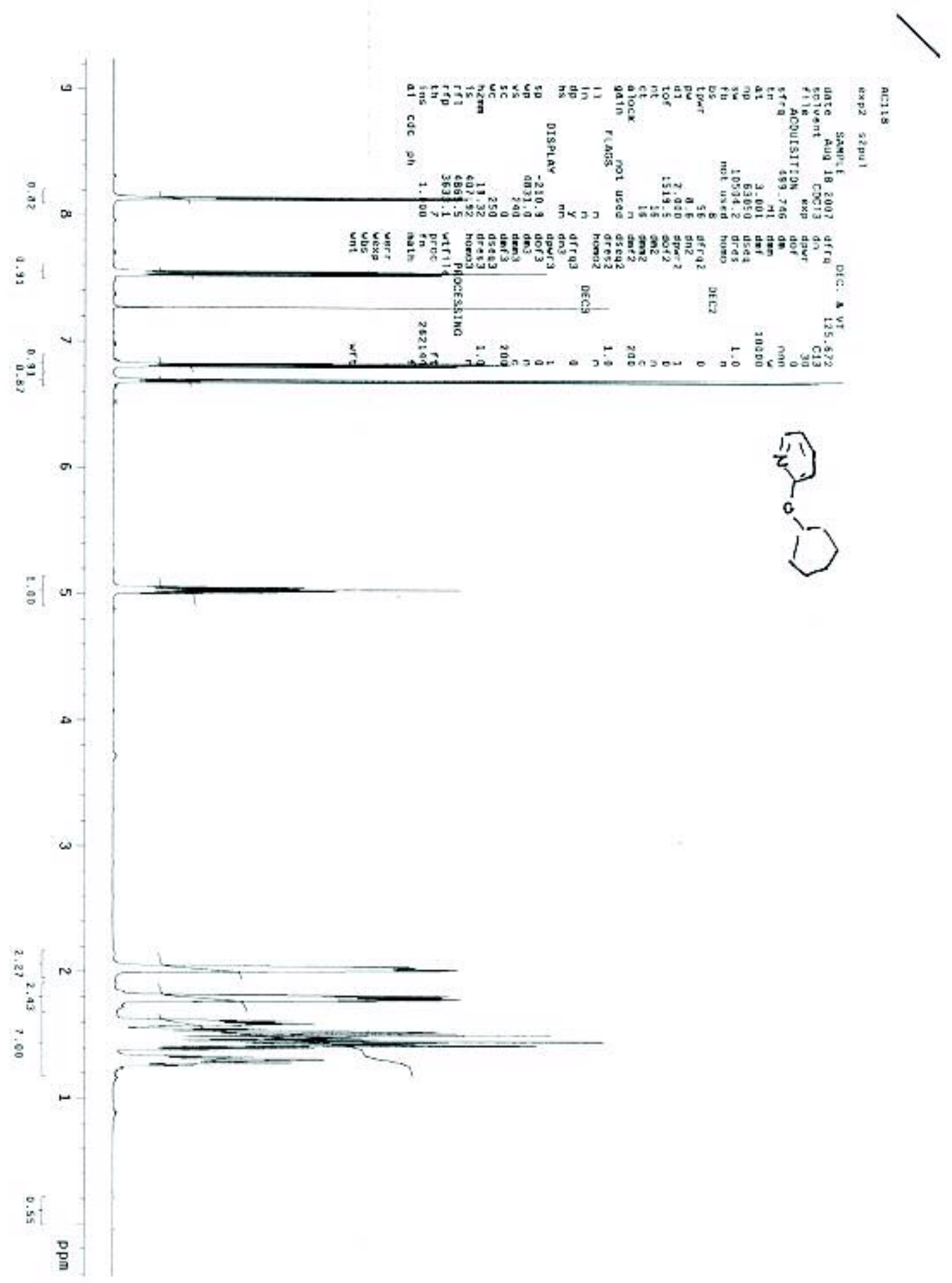




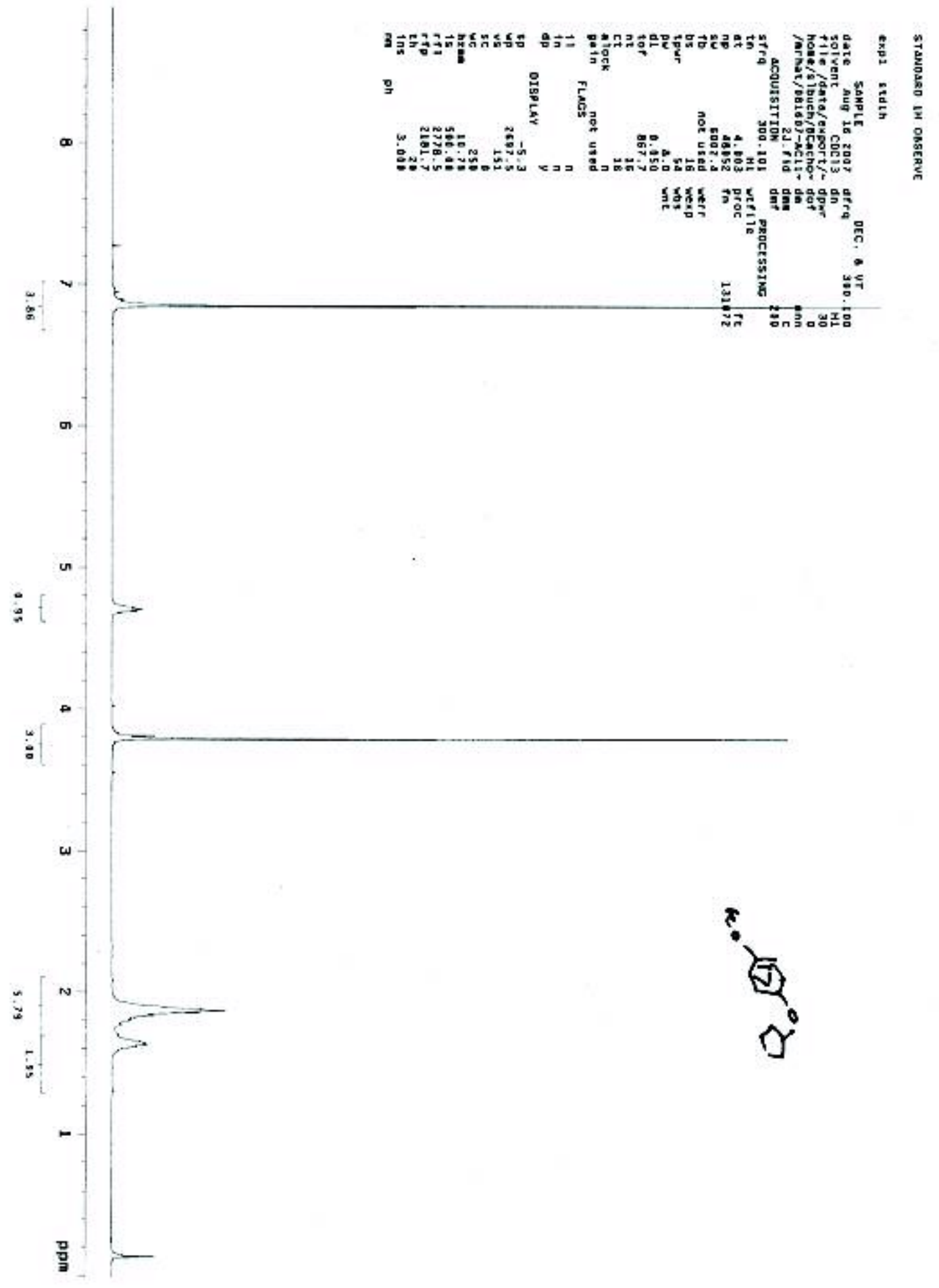

$\stackrel{5}{\Sigma}$ 


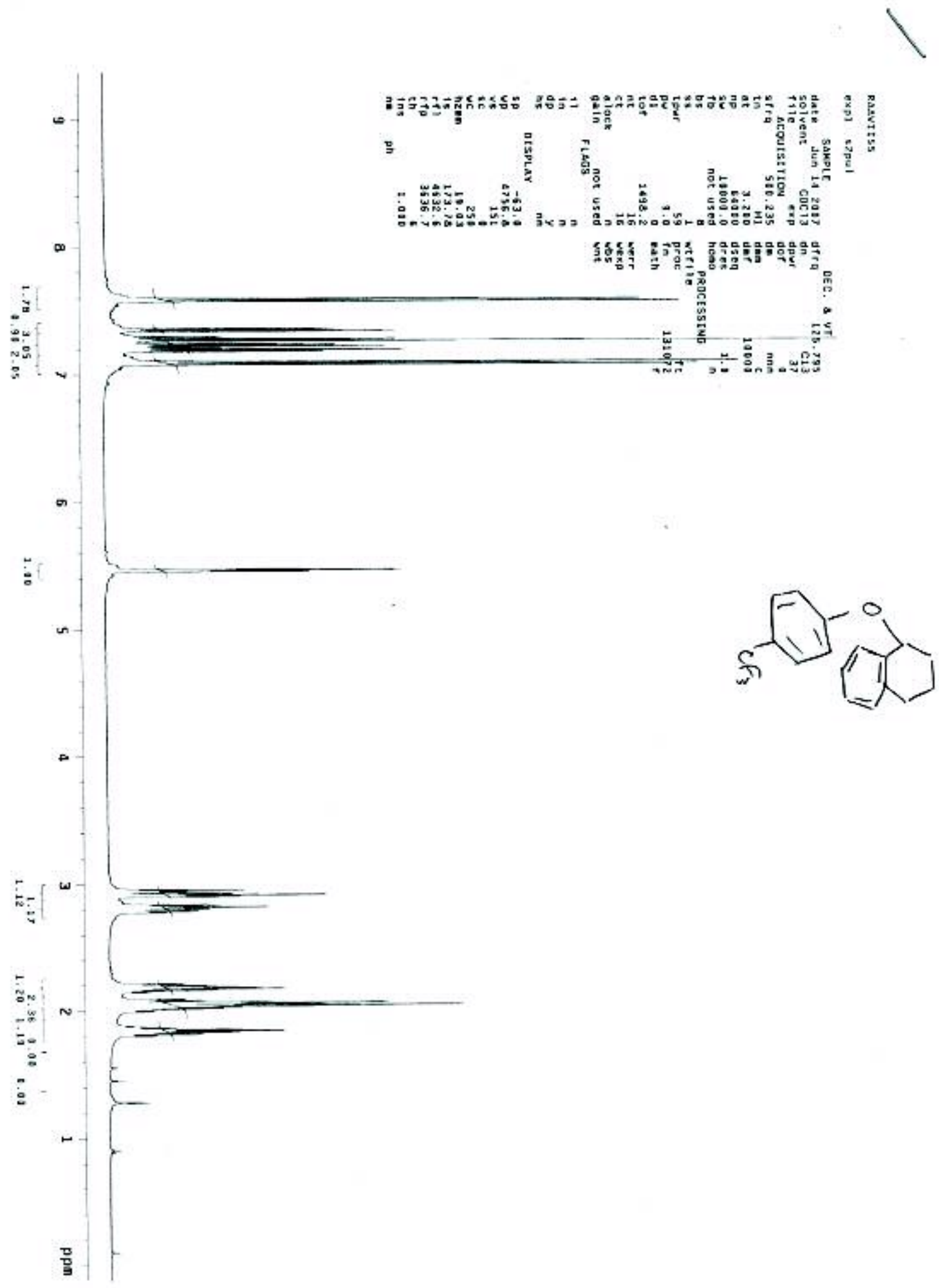




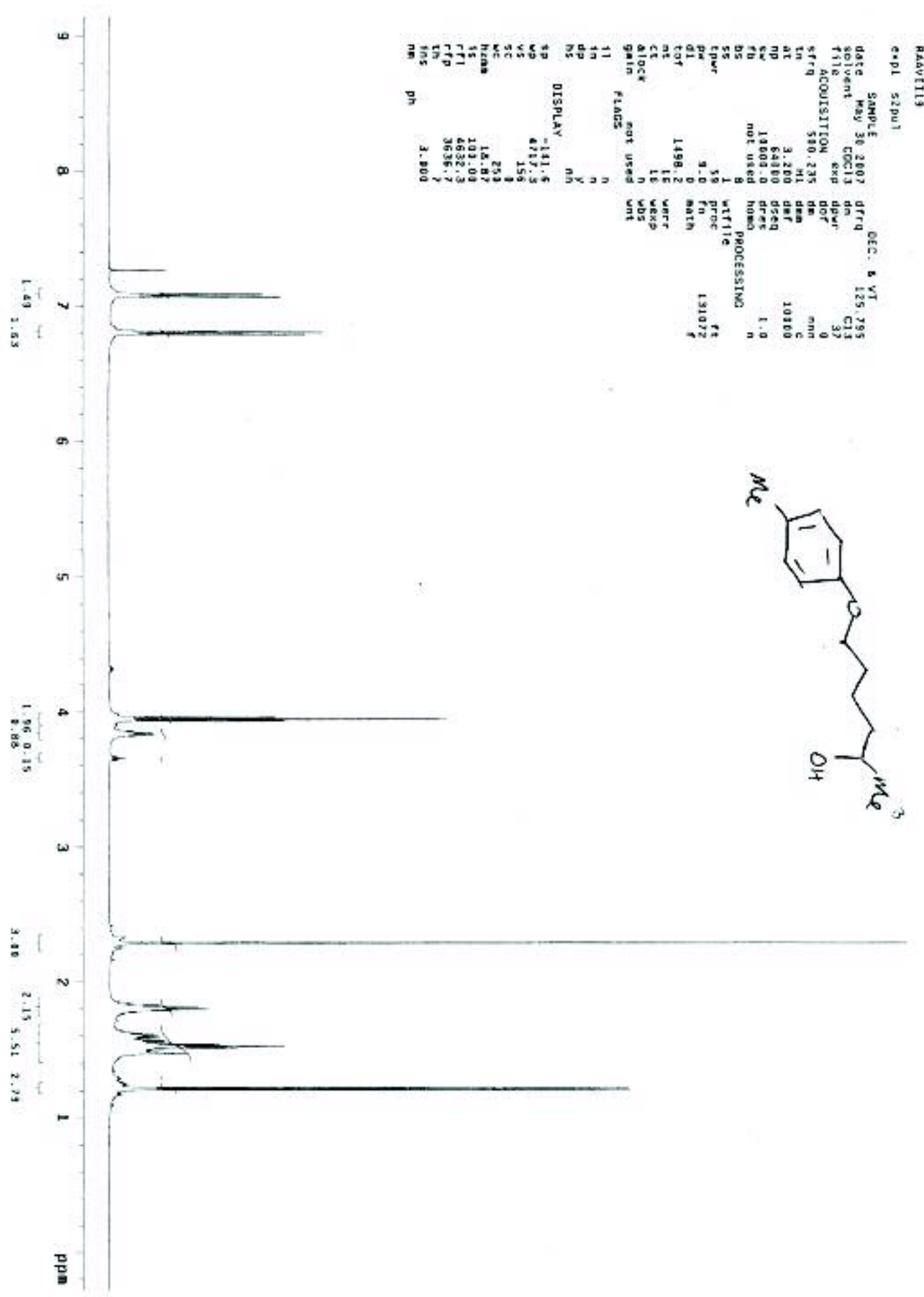




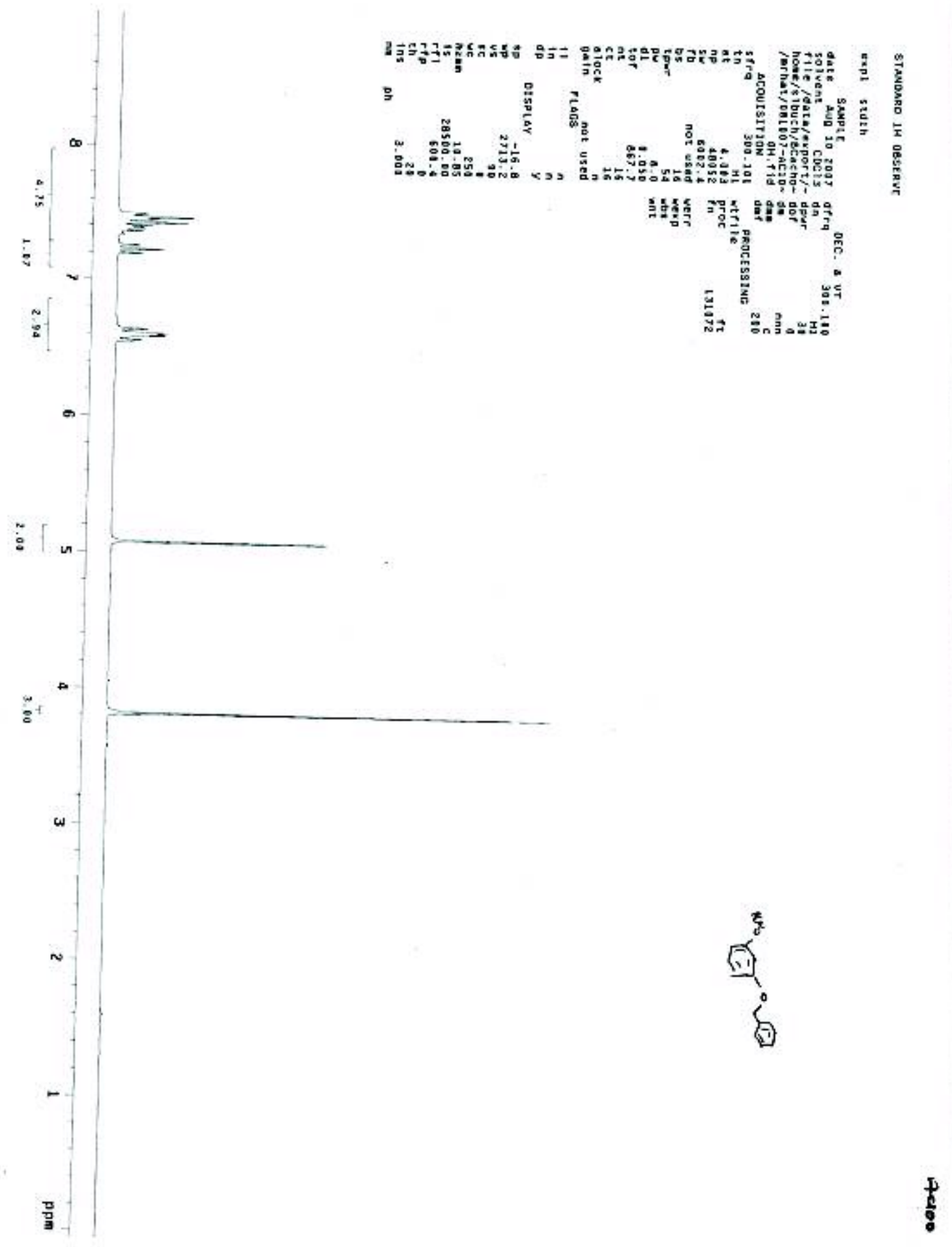




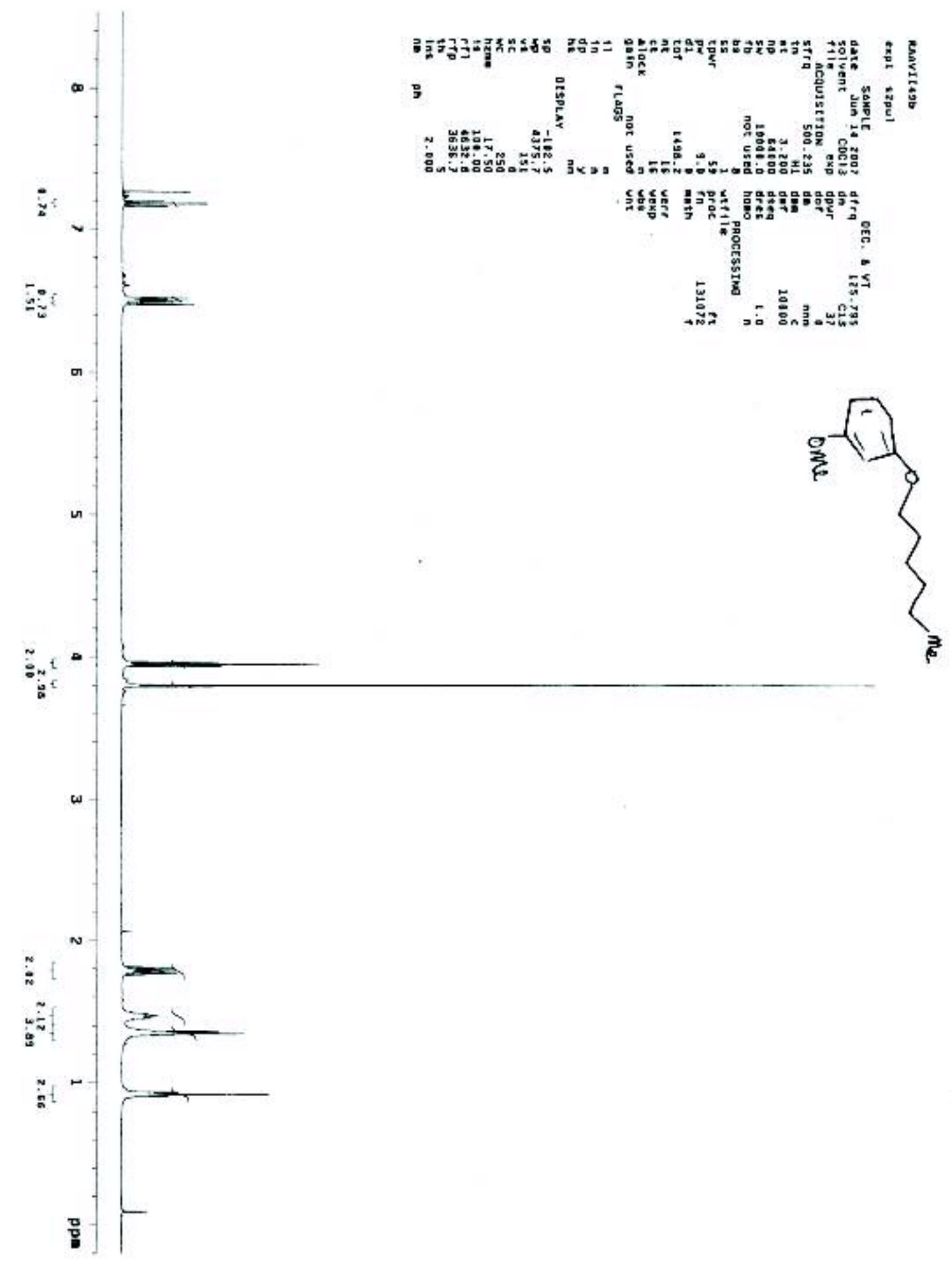




\section{References}

${ }^{\mathrm{i}}$ Stewart, D.; McHattie, G. S.; Imrie, C. T. J. Mater. Chem. 1998, 8, 47 - 52.

ii Dalal et al. J. Indian Chem. Soc. 1958, 35745 - 747.

iii Robbins, T. A.; Knobler, C. B.; Bellew, D. R.; Cram, D. J. J. Am. Chem. Soc. 1994, 116, 111 122.

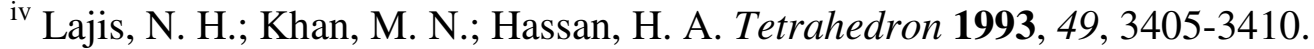

${ }^{v}$ Sharma, G. V. M.; Ilangovan, A.; Sreenivas, P.; Mahalingam, A. K. Syn. Lett. 2000, 615 - 618.

${ }^{v i}$ Chandrasekhar, S.; Prakash, S. J.; Rao, C. L. J. Org. Chem. 2006, 71, 2196 - 2199.

${ }^{\text {vii }}$ Prepared using the procedure described in the following paper: Zhang, Xin-Ge; Zhang, HuiJie; Wang, Zhen; Li, Chao-Xing, Jiegou Huaxue, 2006, 25, 300 - 304.

viii Wolter, M.; Nordmann, G.; Job, G. E.; Buchwald, S. L. Org. Lett. 2002, 4, 973 - 976.

${ }^{\text {ix }}$ Torraca, K. E.; Huang, X.; Parrish, C. A.; Buchwald, S. L. J. Am. Chem. Soc. 2001, 123, 10770 - 10771.

${ }^{x}$ Ruhland, T.; Andersen, K.; Pedersen, H. J. Org. Chem. 1998, 63, 9204 - 9211. 